Geometry 8 Topology

Volume 9 (2005) 757-811

Published: 28 April 2005

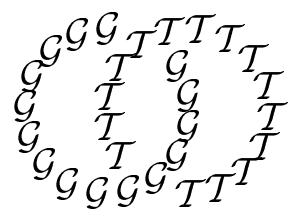

\title{
Complex surface singularities with integral homology sphere links
}

\author{
WaLter D NeUmann \\ JONATHAN WAHL \\ Department of Mathematics, Barnard College, Columbia University \\ New York, NY 10027, USA \\ Department of Mathematics, The University of North Carolina \\ Chapel Hill, NC 27599-3250, USA \\ Email: neumann@math.columbia.edu and jmwahl@email.unc.edu
}

\begin{abstract}
While the topological types of normal surface singularities with homology sphere link have been classified, forming a rich class, until recently little was known about the possible analytic structures. We proved in 30 that many of them can be realized as complete intersection singularities of "splice type," generalizing Brieskorn type.

We show that a normal singularity with homology sphere link is of splice type if and only if some naturally occurring knots in the singularity link are themselves links of hypersurface sections of the singular point.

The Casson Invariant Conjecture (CIC) asserts that for a complete intersection surface singularity whose link is an integral homology sphere, the Casson invariant of that link is one-eighth the signature of the Milnor fiber. In this paper we prove CIC for a large class of splice type singularities.

The CIC suggests (and is motivated by the idea) that the Milnor fiber of a complete intersection singularity with homology sphere link $\Sigma$ should be a 4 -manifold canonically associated to $\Sigma$. We propose, and verify in a non-trivial case, a stronger conjecture than the CIC for splice type complete intersections: a precise topological description of the Milnor fiber.

We also point out recent counterexamples to some overly optimistic earlier conjectures in [28] and [29].
\end{abstract}

AMS Classification numbers Primary: 14B05, 14H20

Secondary: $32 \mathrm{~S} 50,57 \mathrm{M} 25,57 \mathrm{~N} 10$

Keywords: Casson invariant, integral homology sphere, surface singularity, complete intersection singularity, monomial curve, plane curve singularity

Proposed: Robion Kirby

Seconded: Ronald Fintushel, Ronald Stern

Received: 24 May 2004

Revised: 18 April 2005 
In the parallel paper 30 we give analytic descriptions in terms of splice diagrams for a wide range of topologies of singularities, when the link of the singularity is a $\mathbb{Q}$-homology sphere. The splice diagrams considered there generalize the original splice diagrams of [7, 31] in that the numerical weights around a node need not be pairwise coprime. In this paper we restrict to $\mathbb{Z}^{-}$ homology sphere links. Our splice diagrams will thus always have pairwise coprime weights around each node, and, by [7], the possible links are classified by their splice diagrams and are obtained by repeatedly splicing together the links $\Sigma\left(p_{1}, \cdots, p_{n}\right)$ of Brieskorn complete intersections along naturally occurring knots. Even for this restricted class of topologies, only in the simplest cases does one know what analytic properties such singularities might have, eg, being a complete intersection or Gorenstein.

In 30], we describe how "most" homology sphere singularity links arise as links of complete intersection singularities. This occurs when the associated splice diagram satisfies a certain "semigroup condition." In that case we give explicit equations, which we call "splice type," generalizing the Brieskorn complete intersections. One may think in terms of an operation of splicing the defining equations of two singularities which on the boundary corresponds to splicing the links. Specifically, we have the

Theorem 30] Given a homology sphere link $\Sigma$ whose splice diagram satisfies the semigroup condition, there exists a complete intersection singularity of splice type whose link is $\Sigma$.

There is a natural notion of "higher weight terms" for a splice type equation, and, by definition, the result of adding higher weight terms is still of splice typ 1 (the effect on the singularity is always an equisingular deformation). Thus, for example, the splice type singularities corresponding to one-node splice diagrams are precisely the Brieskorn complete intersection singularities with homology sphere link and their higher weight deformations.

In an earlier paper [28, we made the over-optimistic

Splice Type Conjecture Any Gorenstein surface singularity with integral homology sphere link is a complete intersection of splice type.

Implicit in this conjecture was a new necessary condition (the "semigroup condition") on a splice diagram (and hence on a resolution diagram) in order that

\footnotetext{
${ }^{1}$ This differs from 28,29, where higher order terms were not allowed. We now call this "strict splice type."
} 
it come from a Gorenstein singularity. After all, a similar semigroup condition on the value semigroup of a curve singularity is well known to characterize the Gorenstein ones. Further, the conjecture would imply that the topology of a homology sphere link determines a Gorenstein singularity uniquely up to equisingularity - a kind of "tautness." (Compare with the equations of plane curve singularities with given Puiseux pairs.) Indeed, the conjecture is true for any singularity $z^{n}+g(x, y)=0$ with homology sphere link (Corollary 4.2); this is a statement about writing the irreducible $g(x, y)$ in a certain iterative way.

But a class of examples which may be found in the paper [17] of Némethi, Luengo, and Melle-Hernandez, shows this Conjecture to be false in this generality:

Examples (a) There exists a Gorenstein singularity, not of splice type, whose link is the Brieskorn sphere $\Sigma(2,13,31)$.

(b) There exists a Gorenstein singularity, not of splice type, whose link is a homology sphere but which does not satisfy the semigroup conditions.

The above singularities are universal abelian covers of "superisolated" hypersurface singularities. We do not know in either case how to write down equations; in particular, we still know no counterexample to the Splice Type Conjecture for complete intersections.

We prove our original conjecture under additional assumptions, which clarifies the situation. A homology sphere link $\Sigma$ of a normal surface singularity $(X, o)$ has a number of natural knots, one for each leaf of the splice diagram (or equivalently, of the resolution graph). For a splice type singularity these knots are cut out by hyperplane sections. We prove, conversely (see Theorem 4.1 for a more precise version):

Theorem 1 For a normal surface singularity $(X, o)$ with homology sphere link, if all the knots associated to leaves of the splice diagram are links of hypersurface sections of $X$, then the semigroup condition is fulfilled, and $X$ is a complete intersection of splice type.

Our study of singularities with homology sphere link originated in our conjecture, formulated in [26]:

Casson Invariant Conjecture Let $(X, o)$ be an isolated complete intersection surface singularity whose link $\Sigma$ is an integral homology 3-sphere. Then the Casson invariant $\lambda(\Sigma)$ is one-eighth the signature of the Milnor fiber of $X$. 
At the time, we verified the Casson Invariant Conjecture for Brieskorn complete intersections by direct computation. It was a challenge to find other examples, but having done so, the conjecture was verified in these cases, with the serious work being calculation of the signature. With the singularities of splice type we now have an abundance of examples, but even for these the signature calculations are difficult, and we cannot verify the Casson Invariant Conjecture. Still, the following theorem includes all previously proved cases of the Casson Invariant Conjecture, except for some cases described by Collin and Saveliev in [5] (see Remark 8.3).

Theorem 2 The Casson Invariant Conjecture is true for complete intersection singularities of splice type for which the nodes of the splice diagram are in a line.

This is proved by reformulating (as in [26]) the Casson Invariant Conjecture in terms of geometric genus, which is easier than the signature to compute from defining equations.

Casson Invariant Conjecture (Version 2) Let $(X, o)$ be a complete intersection surface singularity with integral homology 3-sphere link $\Sigma$. Then the Casson invariant $\lambda(\Sigma)$ equals $-p_{g}(X, o)-\frac{1}{8} C(\Sigma)$, where $C(\Sigma)$ is the characteristic number $c_{1}^{2}+c_{2}-1$ of any good resolution of $X$ (this is a topological invariant).

This version is equivalent to the previous version by formulas of Laufer and Durfee (see proof of Theorem 6.3. $)$. This formulation makes sense for Gorenstein singularities, but is false in that generality, as seen using some of the examples above.

Assuming the Splice Type Conjecture for complete intersections (a shaky assumption), one might expect to verify the Casson Invariant Conjecture by direct calculation with the equations. But we expect things to go in the opposite direction: a proof of the Casson Invariant Conjecture (perhaps symplectic or gauge-theoretic) might help deduce the form of defining equations. This happens for instance in the one-node case: we proved in 26] that a Gorenstein singularity $(X, o)$ with link $\Sigma\left(p_{1}, \cdots, p_{n}\right)$ is of splice type, ie, an equisingular deformation of the corresponding Brieskorn complete intersection, if and only if the Casson Invariant Conjecture holds for $X$ (equivalently, $X$ has the same geometric genus as the Brieskorn complete intersection). We remark that A. Némethi [19] has proved this value of geometric genus for weakly elliptic singularities, eg, when the link is $\Sigma(2,3,6 k+5)$. 
Part of the interest of the Casson Invariant Conjecture is its suggestion that the Milnor fiber is a "natural" 4-manifold which is attached to its boundary $\Sigma$, and for which the signature computes the Casson invariant exactly (and not just mod 2). Specifically, it implies that for a complete intersection singularity whose link is a homology sphere, analytic invariants like the Milnor number and geometric genus are determined by the link. (Such results are known to be false for general hypersurface singularities.) Given the equations of a singularity, it is relatively easy to calculate the Casson invariant of the link, but it is extremely hard to calculate the signature of the Milnor fiber (let alone understand its topology).

We conjecture a topological construction that, when splicing two singularities, creates the new Milnor fiber out of the old ones, extending the operation of splicing on the boundaries (see Conjecture 2). This conjecture easily implies the Casson Invariant Conjecture for splice type singularities (Corollary [6.2). We succeed in proving it in a non-trivial case:

Theorem 3 For a singularity $z^{n}+g(x, y)=0$ with homology sphere link, the Milnor fiber is formed by the conjectured topological construction.

Though the Casson Invariant Conjecture for this case follows, it had already been proven in [26] (by a much less conceptual proof), and more recently by Collin and Saveliev [5] using equivariant Casson invariants and by Némethi and Nicolaescu 23] in a more general context. It is also a special case of Theorem [2]

In [28, 29] we proposed a more general version of the Splice Type Conjecture: Any $\mathbb{Q}$-Gorenstein surface singularity with $\mathbb{Q}$-homology sphere link has as universal abelian cover a complete intersection singularity of splice type (using a more general notion of splice diagram). Although true surprisingly often, the examples of [17] mentioned above show this to be false in general, even for hypersurface singularities.

The converse direction, that equations of splice type lead to abelian covers of $\mathbb{Q}$-Gorenstein singularities with expected topological type, is the main content of [30] (in particular, as already mentioned, the equations of splice type of the current paper give singularities with the expected homology sphere links). This paper is nevertheless somewhat transverse to [30, since we offer there no guess as to the topology or the signature of the Milnor fiber of the universal abelian cover. Though [26] wondered about a generalization of the Casson Invariant Conjecture for $\mathbb{Q}$-homology sphere links involving the Casson-Walker invariant, computations for Seifert fibered rational homology spheres by Lescop [15, 16] 
showed the naive generalization fails (see also [4]). Lim's result [14 suggested looking at a Seiberg-Witten invariant, and a recent generalization along these lines of the Casson Invariant Conjecture to $\mathbb{Q}$-Gorenstein $\mathbb{Q}$-homology spheres has been offered by Némethi and Nicolaescu [21, 22, 23, but is now also known to be false in the generality stated (see [17]).

We offer now a road map to help readers go through this paper.

Sections 1 and 2 are introductory. In Section 1] we review from [7] the definition of splice diagrams and the topological description of homology sphere links; further details are found in the Appendix (Section 91), where we also give an improved description of the relationship between splice diagrams and plumbing (or resolution) graphs. We also introduce the important "semigroup condition." In Section 2 we associate "splice type equations" to any splice diagram with semigroup condition; this provides a wealth of examples of complete intersections with homology sphere links. Modifying the construction provides familiar equations for complete intersection monomial curves.

Section [3 develops some theory of semigroups and monomial curves that is needed in the next two sections to prove Theorems 1 and 2. In particular, it includes a new characterization of complete intersection monomial curves in terms of one-dimensional analogues of splice type singularities (Theorem 3.1] and its scholium).

Section 4 examines the key property of a splice type singularity: the natural knots in the link associated to leaves in the splice diagram are obtained by setting a coordinate equal to 0 . We prove (Theorem 4.1, a more precise version of Theorem (1) that conversely any normal surface singularity with homology sphere link, and for which the natural knots are hypersurface sections, is in fact a splice type singularity. Major use is made of Theorem 3.1 concerning the $\delta$-invariant of certain monomial curves.

Section 5 has as its goal the inductive calculation of the geometric genus $p_{g}$ for a splice type singularity. Every node $v$ of the splice diagram gives a valuation (or weight function) $\nu$ of the singularity; a key result (Theorem 5.3) states that the associated graded ring associated to $\nu$ is an integral domain, whose normalization is a Brieskorn complete intersection. Now, $p_{g}$ is the colength of the "canonical ideal," given by functions for which every $\nu$-weight is at least some explicit value. When all the nodes of the splice diagram are on a line, there is a simultaneous monomial basis for every associated graded (Lemma 5.5). This reduces the calculation of $p_{g}$ in that case to counting integral lattice points in some region; an induction now works, yielding the main result, Theorem 5.6. Theorem 2 is a corollary of this and Theorem 6.3 of the next section. 
The remaining sections [6 to 8 discuss the Milnor Fiber Conjecture and are largely independent of the preceding sections. Section 6 introduces this conjecture, which describes the conjectured topology of the Milnor fiber of splice type singularities, and which would imply the Casson Invariant Conjecture. The discussion leads to Theorem 6.3. which clarifies how the Casson Invariant Conjecture relates to splicing. This involves the relationship between signature and geometric genus, and the key is to understand the behavior of the topological invariant $C(\Delta)$ of the link under splicing. This is done in Theorem 6.4 whose proof, using numerics of splice diagrams, takes up the following section (Section 7).

Section 8 verifies the Milnor Fiber Conjecture for equations of the form $z^{n}=$ $f(x, y)$, by careful topological construction of the Milnor fiber. This uses a description of plane curve singularities in terms of splice diagram equations.

Acknowledgements The conjectures and some results of this paper arose from a visit by the first author to Duke University, and we thank the Duke Mathematics Department for its hospitality. We also thank the Max-PlanckInstitut für Mathematik in Bonn for its hospitality while some of the work on this paper was done.

The first author's research is supported under NSF grant DMS-0083097 and the second author's under NSA grant MDA904-02-1-0068.

\section{Splice diagrams for integral homology sphere links}

For more details on splicing see the Appendix (Section 9).

Recall that a splice diagram is a finite tree with vertices only of valency 1 ("leaves") or $\geq 3$ ("nodes") and with a collection of integer weights at each node, associated to the edges departing the node. The following is an example.

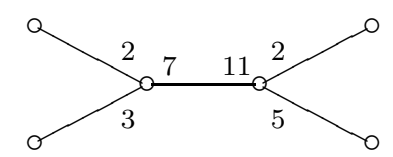

For an edge connecting two nodes in a splice diagram the edge determinant is the product of the two weights on the edge minus the product of the weights adjacent to the edge. Thus, in the above example, the one edge connecting two nodes has edge determinant $77-60=17$.

The splice diagrams that classify homology sphere singularity links satisfy the following conditions on their weights: 
- the weights around a node are positive and pairwise coprime;

- the weight on an edge ending in a leaf is $>1$;

- all edge determinants are positive.

More general splice diagrams appear for other situations (see, eg, 7, 29, 30]), but we will only consider splice diagrams satisfying the above conditions here.

Theorem 1.1 7] The homology spheres that are singularity links are in oneone correspondence with splice diagrams satisfying the above conditions.

The splice diagram and resolution diagram for the singularity determine each other uniquely, and describe how to construct the link by splicing or by plumbing. One method to compute the resolution diagram from the splice diagram is given in [7. We describe an easier method in the appendix to this paper (Section 9), where we also recall the topological meaning of splicing and how to compute the splice diagram from the resolution diagram for a singularity.

The following notations will be used extensively in this paper.

Notation For a node $v$ and an edge $e$ at $v$, let $d_{v e}$ be the weight on $e$ at $v$, and $d_{v}$ the product of the $d_{v e}$ over all such $e$. Let $\Delta_{v e}$ be the subgraph of $\Delta$ cut off from $v$ by $e$. For any pair of vertices $v$ and $w$, let $\ell_{v w}$ (the linking number) be the product of all the weights adjacent to, but not on, the shortest path from $v$ to $w$ in $\Delta$. We also consider $\ell_{v w}^{\prime}$, the same product but excluding weights around $v$ and $w$. Thus if $v$ is a node and $w$ is a leaf in $\Delta_{v e}$, then

$$
\ell_{v w} d_{v e}=\ell_{v w}^{\prime} d_{v} .
$$

Definition 1.2 Semigroup Condition Let $\Delta$ be a splice diagram. We say $\Delta$ satisfies the semigroup condition if, for each node $v$ and adjacent edge $e$, the edge-weight $d_{v e}$ is in the semigroup

$$
\mathbb{N}\left\langle\ell_{v w}^{\prime}: w \text { a leaf of } \Delta \text { in } \Delta_{v e}\right\rangle .
$$

Equivalently, the product $d_{v}$ of the edge-weights adjacent to $v$ is in the semigroup

$$
\mathbb{N}\left\langle\ell_{v w}: w \text { a leaf of } \Delta \text { in } \Delta_{v e}\right\rangle .
$$

For instance, in the two-node splice diagram above, let $v$ be the leftmost node and $w$ the upper right hand leaf. Then $\ell_{v w}$ equals $2 \cdot 3 \cdot 5$, while $\ell_{v w}^{\prime}=5$; the semigroup condition is satisfied at that node since 7 is in the semigroup generated by 2 and 5 . 
If a splice diagram satisfies the semigroup condition, we will write down complete intersection equations that give a singularity with the given link. We know of no counterexample to the following optimistic conjecture mentioned in [29].

Conjecture 1 (Complete Intersection implies Semigroup Condition) If a surface singularity with homology sphere link is a complete intersection, then its splice diagram satisfies the semigroup condition.

For example, consider the splice diagram

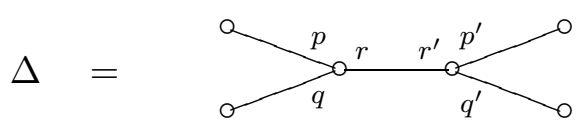

with $p, q, r$ and $p^{\prime}, q^{\prime}, r^{\prime}$ pairwise coprime triples of positive integers satisfying $r r^{\prime}>p q p^{\prime} q^{\prime}$. Then $\Delta$ satisfies the semigroup condition if and only if

$$
r \in \mathbb{N}\left\langle p^{\prime}, q^{\prime}\right\rangle \quad \text { and } \quad r^{\prime} \in \mathbb{N}\langle p, q\rangle \text {. }
$$

(Note $r$ is automatically in the semigroup $\mathbb{N}\left\langle p^{\prime}, q^{\prime}\right\rangle$ if it is greater than or equal to the conductor $\left(p^{\prime}-1\right)\left(q^{\prime}-1\right)$.) In particular, the resolution diagram

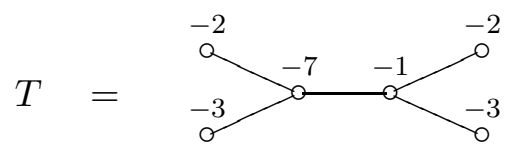

gives the splice diagram

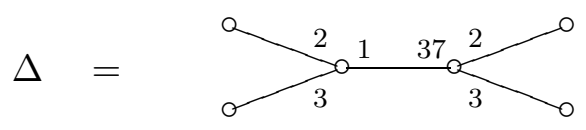

which does not satisfy the semigroup condition, since 1 is not in the semigroup generated by 2 and 3. We would therefore expect that there is no complete intersection singularity with this resolution.

\section{Equations associated to a splice diagram}

Let $\Delta$ be a splice diagram satisfying the semigroup condition. We will write down a system of complete intersection equations that give a singularity with the corresponding link. Associate a variable $z_{w}$ to each leaf $w$ of the splice diagram. To each node $v$ of the splice diagram, we will associate $\left(\delta_{v}-2\right)$ equations, where $\delta_{v}$ is the valency of the node. If $n$ is the number of leaves, 
then it is easy to check that $n-2=\sum\left(\delta_{v}-2\right)$ (summed over the nodes of $\Delta$ ), so this will give the right number of equations.

Fix a node $v$. For each leaf $w$ we give the variable $z_{w}$ weight $\ell_{v w}$ (we call this the $v$-weight of $z_{w}$ ). For each edge $e$ at $v$ the semigroup condition lets us write

$$
d_{v}=\sum_{w} \alpha_{v w} \ell_{v w}, \quad \text { sum over the leaves } w \text { of } \Delta \text { in } \Delta_{v e}, \text { with } \alpha_{v w} \in \mathbb{N} \text {. }
$$

Equivalently,

$$
d_{v e}=\sum_{w} \alpha_{v w} \ell_{v w}^{\prime}, \quad \text { sum over the leaves } w \text { of } \Delta \text { in } \Delta_{v e}
$$

We define an admissible monomial (associated to the edge $e$ at the node $v$ ) to be a monomial $\prod_{w} z_{w}^{\alpha_{v w}}$, the product over leaves $w$ in $\Delta_{v e}$, with exponents satisfying the above equations. Thus an admissible monomial $M_{v e}$ associated to $v$ has total $v$-weight $d_{v}$ (and depends on the choice of $\alpha_{v w}$ ).

Next, choose one admissible monomial $M_{v e}$ for each edge at $v$ and consider $\delta_{v}-2$ equations associated to $v$ by equating to 0 some $\mathbb{C}$-linear combinations of these monomials:

$$
\sum_{e} a_{i e} M_{v e}=0, \quad i=1, \ldots, \delta_{v}-2 .
$$

Repeating for all nodes, we get a total of $n-2$ equations. If the coefficients $a_{i e}$ of the equations are "sufficiently general," we say that the resulting system of $n-2$ equations is of strict splice type.

Sufficiently general simply means that for every $v$, all maximal minors of the $\left(\delta_{v}-2\right) \times \delta_{v}$ matrix $\left(a_{i e}\right)$ of coefficients should be non-singular. By applying row operations to such a matrix (taking linear combinations of the equations) one can always put the $\left(\delta_{v}-2\right) \times \delta_{v}$ coefficient matrix in the form

$$
\left(\begin{array}{cccccc}
1 & 0 & \ldots & 0 & a_{1} & b_{1} \\
0 & 1 & \ldots & 0 & a_{2} & b_{2} \\
\vdots & \vdots & & \vdots & \vdots & \vdots \\
0 & 0 & \ldots & 1 & a_{\delta_{v}-2} & b_{\delta_{v}-2}
\end{array}\right)
$$

so we will often assume we have done so. In this way, the defining equations are sums of three monomials. The "sufficiently general" condition is then $a_{i} b_{j}$ $a_{j} b_{i} \neq 0$ for all $i \neq j$, and all $a_{i}$ and $b_{i}$ nonzero. 
Example 1 Assume $\Delta$ has one node, of valency $n$. There is no semigroup condition. There is only one admissible monomial for each edge, namely $z_{j}^{d_{j}}$, where $d_{j}$ is the weight on the edge. Our equations are thus of Brieskorn type:

$$
\sum_{j=1}^{n} a_{i j} z_{j}^{d_{j}}=0, \quad i=0, \ldots, n-2 .
$$

The "sufficiently general" condition is then the well-known condition (due to H. Hamm [9]) for the system of $n-2$ equations to have an isolated singularity. Thus, for a splice diagram with one node, "strict splice type" is equivalent to isolated Brieskorn complete intersection.

Example 2 For the $\Delta$ of the example at the start of Section 1 we associate variables $z_{1}, \ldots, z_{4}$ to the leaves as follows:

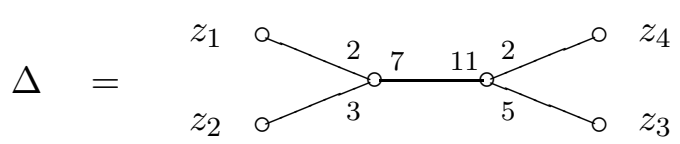

The admissible monomials for the left node are $z_{1}^{2}, z_{2}^{3}$, and $z_{3} z_{4}$. The admissible monomials for the right node are $z_{3}^{5}, z_{4}^{2}$, and $z_{1} z_{2}^{4}$ or $z_{1}^{3} z_{2}$ (since $11=\alpha \cdot 3+\beta \cdot 2$ has solutions $(1,4)$ and $(3,1))$. Thus the system of equations might be

$$
\begin{aligned}
& z_{1}^{2}+z_{2}^{3}+z_{3} z_{4}=0, \\
& z_{3}^{5}+z_{4}^{2}+z_{1} z_{2}^{4}=0 .
\end{aligned}
$$

This system is always of "strict splice type" by our comments above.

Equisingular deformations of systems of equations of strict splice type should come from adding terms of greater or equal weight with respect to the vertex weights to each equation. If only greater weight is allowed the result always is an equisingular deformation. We speak of a higher weight deformation and say the resulting equations are simply of splice type. See [30] for a fuller discussion.

The importance of splice type singularities is indicated by a result:

Theorem 2.1 30] A system of equations of splice type defines an isolated complete intersection surface singularity whose link is the homology sphere $\Sigma$ defined by the splice diagram $\Delta$, and whose resolution graph is therefore the corresponding resolution diagram.

Each node $v$ of the splice diagram corresponds to an exceptional curve $E_{v}$ of the resolution, and the $v$-weight of $z_{i}$ is its value for the valuation given by order of vanishing on $E_{v}$. 
Moreover, the curve $z_{i}=0$ cuts out in $\Sigma$ the knot corresponding to the $i$-th leaf of $\Delta$.

One could expand the definition of strict splice type singularities to include (for fixed $v$ ) suitable linear combinations of all possible admissible monomials associated to edges at $v$. But, up to higher weight deformations, this adds no generality. Also, if we change our choice of admissible monomials for the edges at each node, then we only change our splice type singularities up to higher weight deformation. Thus the concept of splice type is independent of choices of admissible monomials.

The theorem implies that the embedding dimension of a splice diagram singularity is at most the number of leaves of the splice diagram - but it may be less. There are even unexpected hypersurface examples.

Example 3 Let $\Delta$ be the splice diagram:

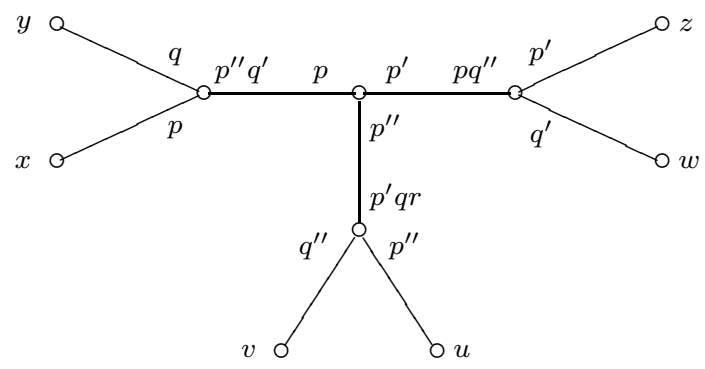

The integers $p, q, p^{\prime}, q^{\prime}, p^{\prime \prime}, q^{\prime \prime}, r$ are $\geq 2$ and satisfy appropriate relative primeness conditions, as well as edge inequalities

$$
q^{\prime}>p^{\prime} q, \quad q^{\prime \prime}>p^{\prime \prime} q^{\prime}, \quad q r>p q^{\prime \prime} .
$$

Associating variables $x, y, z, w, u, v$ to the leaves in clockwise order starting from the left as shown, one may write splice equations:

$$
\begin{aligned}
x^{p}+y^{q} & =z \\
z^{p^{\prime}}+w^{q^{\prime}} & =u \\
u^{p^{\prime \prime}}+v^{q^{\prime \prime}} & =x^{r} \\
y+w & =v
\end{aligned}
$$

These define the hypersurface singularity given by

$$
\left(\left(x^{p}+y^{q}\right)^{p^{\prime}}+w^{q^{\prime}}\right)^{p^{\prime \prime}}+(y+w)^{q^{\prime \prime}}=x^{r} .
$$


A variant of the splice diagram yields a more familiar object. Let $\Delta$ be a splice diagram satisfying the semigroup conditions, and choose a distinguished leaf $w^{\prime}$, to form a "rooted diagram." Attach a variable $z_{w}$ to each leaf $w \neq$ $w^{\prime}$. Now, for each vertex $v$ of $\Delta$, form the same equations as before, except that one does not consider the edge in the direction of $w^{\prime}$. (One is in general eliminating more monomials than simply setting $z_{w^{\prime}}=0$ in our previous splice diagram equations.) There is now one equation less than there are variables. Note that the edge-weights in the direction of $w^{\prime}$ now play no role and can be discarded. We claim these equations generate a complete intersection curve, and this curve is the monomial curve associated to a semigroup $\Gamma^{\prime}$. To describe this we first briefly recall some terminology about semigroups (see Section 3 for more details).

The semigroups arising in this paper are always numeric semigroups, that is subsemigroups $\Gamma$ of $\mathbb{N}=\mathbb{Z}_{\geq 0}$ for which $\mathbb{N}-\Gamma$ is finite. The conductor $c(\Gamma)$ is the smallest $c \geq 0$ so that $\gamma \geq c$ implies $\gamma \in \Gamma$. The semigroup ring $\mathbb{C}\left[t^{\Gamma}\right]$, or monomial curve associated to $\Gamma$, is the graded subalgebra of $\mathbb{C}[t]$ generated by $t^{\gamma}, \gamma \in \Gamma$. $\Gamma$ is called a complete intersection semigroup if $\mathbb{C}\left[t^{\Gamma}\right]$ is a graded complete intersection.

In our situation of a splice diagram $\Delta$ satisfying the semigroup conditions with distinguished leaf $w^{\prime}$, the semigroup $\Gamma^{\prime}$ is the semigroup generated by $\ell_{w^{\prime} w}$ over all leaves $w \neq w^{\prime}$. We will see in section [3 that:

Fact $\Gamma^{\prime}$ is a complete intersection semigroup and the modified splice equations described above define the monomial curve $\mathbb{C}\left[t^{\Gamma^{\prime}}\right]$.

In terms of Theorem 2.1 the significance of this curve is that if $w^{\prime}$ is the $i$-th leaf of $\Delta$ then this curve, or an equisingular deformation of it, arises as the curve cut out by the hyperplane $z_{i}=0$.

Example 4 Consider the splice diagram at the beginning of Section 1 and let $w^{\prime}$ be the lower left leaf. In the modified splice diagram, the weights 3 and 11 are removed. Denote the three leaves by $w_{i}, i=1,3,4$, starting at the upper left and going counterclockwise; the corresponding variables by $z_{i}$; and the two nodes by $v$ and $v^{\prime}$. Then the equations at $v$ resp. $v^{\prime}$ could be $z_{1}^{2}+a z_{3} z_{4}=0$ and $z_{3}^{5}+b z_{4}^{2}=0$. The semigroup $\Gamma^{\prime}$ is $\Gamma^{\prime}=\mathbb{N}\langle 7,4,10\rangle$ and, if we choose $a=b=-1$, the curve can be parametrized as $\left(z_{1}, z_{3}, z_{4}\right)=\left(t^{7}, t^{4}, t^{10}\right)$.

A leaf $w^{\prime}$ of a splice diagram $\Delta$ always represents a knot in the corresponding homology sphere, and this knot is a fibered knot (see Section 11 of [7]). If the 
homology sphere is given as a link of a splice type singularity as above, then this knot is the link of the curve cut out by a coordinate hyperplane $z_{i}=0$ (and the fibration can be given by the usual Milnor fibration $z_{i} /\left|z_{i}\right|$ ). The first Betti number of its fiber is the Milnor number of the knot. We recall that even without the semigroup condition, we have:

Theorem 2.2 [7, Section 11] The Milnor number of the above knot is

$$
1+\sum_{v \neq w^{\prime}}\left(\delta_{v}-2\right) \ell_{v w^{\prime}}
$$

If the link is given by splice type equations, then the theory of curve singularities implies that this number equals the conductor of the above semigroup $\Gamma^{\prime}$, as can be confirmed by computation of the conductor (Theorem 3.1).

\section{$3 \quad$ Numerical semigroups and monomial curves}

In this section we develop some results about semigroups and their associated curves that are needed in the proofs of Theorems 1 and 2 of the Introduction.

As mentioned in Section 2 the semigroups we consider are always numeric semigroups, that is, subsemigroups $\Gamma$ of $\mathbb{N}=\mathbb{Z}_{\geq 0}$ for which $\mathbb{N}-\Gamma$ is finite. The semigroup ring $\mathbb{C}\left[t^{\Gamma}\right]$, or monomial curve associated to $\Gamma$, is the graded subalgebra of $\mathbb{C}[t]$ generated by $t^{\gamma}, \gamma \in \Gamma$. We briefly collect some known facts and terminology (eg, [6, 10, 11, 35]).

The conductor $c(\Gamma)$ is the smallest $c \geq 0$ so that $\gamma \geq c$ implies $\gamma \in \Gamma$. $\Gamma$ is symmetric when $\gamma \in \Gamma$ if and only if $c(\Gamma)-1-\gamma \notin \Gamma$; equivalently, $\mathbb{C}\left[t^{\Gamma}\right]$ is Gorenstein (see [1] Prop. 2.21). Since $\gamma$ and $c(\Gamma)-1-\gamma$ cannot both be in $\Gamma$, a symmetric semigroup is maximal with given conductor. Classically an element of $\mathbb{N}$ that is not in $\Gamma$ is called a gap. The number of gaps is denoted $\delta(\Gamma)$; clearly

$$
\delta(\Gamma) \geq c(\Gamma) / 2, \quad \text { with equality if and only if } \Gamma \text { is symmetric. }
$$

$\Gamma$ is called a complete intersection semigroup if $\mathbb{C}\left[t^{\Gamma}\right]$ is a graded complete intersection. A complete intersection semigroup is symmetric. $\Gamma$ is a complete intersection semigroup if and only if it has a semigroup presentation of deficiency one (ie, with one fewer relations than generators; see [10]). If $\Gamma$ (complete intersection or not) has a semigroup presentation

$$
\Gamma=\left\langle x_{1}, \ldots, x_{n}: \sum_{j} a_{i j} x_{j}=\sum_{j} b_{i j} x_{j}, i=1, \ldots, r\right\rangle
$$


with $a_{i j}, b_{i j} \in \mathbb{N}$, then the monomial curve is presented as

$$
\mathbb{C}\left[z_{1}, \ldots, z_{n}\right] /\left(\prod_{j} z_{j}^{a_{i j}}-\prod_{j} z_{j}^{b_{i j}}, i=1, \ldots, r\right) .
$$

Example Relatively prime $p$ and $q$ generate a complete intersection semigroup with conductor $(p-1)(q-1)$. This semigroup has semigroup presentation $\left\langle x_{1}, x_{2}: q x_{1}=p x_{2}\right\rangle$. Its monomial curve $\mathbb{C}\left[t^{p}, t^{q}\right]$ is presented as $\mathbb{C}\left[z_{1}, z_{2}\right] /\left(z_{1}^{q}-z_{2}^{p}\right)$, with the isomorphism given by $z_{1} \mapsto t^{p}, z_{2} \mapsto t^{q}$.

Let $\left(\Delta, w^{\prime}\right)$ be a finite rooted tree (tree with one vertex singled out as "root"), whose root vertex $w^{\prime}$ is of valency 1 . We visualize it with the root vertex at the top, so "downward" means in the direction away from the root. We assume also that $\Delta$ has positive integer weights on all edges other than the root edge and that the weights on the downward edges at each non-root vertex are pairwise coprime. For example, one obtains such a tree if one picks some leaf $w^{\prime}$ of a splice diagram as root, and then forgets all "far weights" of the edges of the splice diagram (from the point of view of $w^{\prime}$ ); equivalently, one forgets the "near weights" around each node.

In such a tree, the numbers $\ell_{w^{\prime} v}$ for $v \neq w^{\prime}$ are still defined (product of weights on edges directly adjacent to the shortest path from $w^{\prime}$ to $v$ ). We define the semigroup of $\left(\Delta, w^{\prime}\right)$ to be the semigroup

$$
\operatorname{sg}(\Delta)=\operatorname{sg}\left(\Delta, w^{\prime}\right):=\mathbb{N}\left\langle\ell_{w^{\prime} w}: w \text { is a leaf of } \Delta\right\rangle
$$

(we use the shorter $\operatorname{sg}(\Delta)$ if the root vertex is clear). Each non-root vertex of $\Delta$ cuts off a collection of subtrees below it. We say that $\left(\Delta, w^{\prime}\right)$ satisfies the semigroup condition if the weight on the root edge of every such subtree is in the semigroup of the subtree.

Define an invariant $\mu\left(\Delta, w^{\prime}\right)$ by

$$
\mu(\Delta)=\mu\left(\Delta, w^{\prime}\right):=1+\sum_{v \neq w^{\prime}}\left(\delta_{v}-2\right) \ell_{w^{\prime} v} .
$$

Theorem 3.1 Let $\left(\Delta, w^{\prime}\right)$ be a weighted rooted tree as above and $\Gamma=\operatorname{sg}(\Delta)$. Then

$$
2 \delta(\Gamma) \leq \mu(\Delta),
$$

with equality if and only if $\left(\Delta, w^{\prime}\right)$ satisfies the semigroup condition, in which case $\Gamma=\operatorname{sg}(\Delta)$ is a complete intersection semigroup. (It follows that the same result holds with $2 \delta(\Gamma)$ replaced by $c(\Gamma)$.) 
If $\left(\Delta, w^{\prime}\right)$ satisfies the semigroup condition we will describe the complete intersection equations; these equations will be associated to the nodes of $\Delta$. We assign a variable $z_{j}$ to each leaf $w_{j}$ of $\Delta$. The equations will generate the kernel of the map $\mathbb{C}\left[z_{1}, \ldots, z_{m}\right] \rightarrow \mathbb{C}\left[t^{\Gamma}\right]$ given by $z_{j} \mapsto t^{l^{\prime} w_{j}}$.

For a node $v$ of the tree and a leaf $w_{j}$ below it let $l_{v w_{j}}^{\prime}$ be the product of weights adjacent to the path from $v$ to $w_{j}$, excluding weights adjacent to $v$. For each downward edge $e$ at $v$ the semigroup condition tells us that the weight $p_{e}$ is a non-negative integer linear combination $p_{e}=\sum_{j} \alpha_{j} l_{v w_{j}}^{\prime}$, summed over the leaves below $v$. We choose such an expression and denote by $M_{e}=\prod_{j} z_{j}^{\alpha_{j}} \in$ $\mathbb{C}\left[z_{1}, \ldots, z_{m}\right]$ the corresponding monomial. Then:

Scholium If $\left(\Delta, w^{\prime}\right)$ satisfies the semigroup condition in the above theorem then the equations associated to node $v$ are the equations that equate the monomials $M_{e}$ for the different downward edges at $v$.

If we replace each of these equations $M_{e}=M_{e^{\prime}}$ by an equation $M_{e}=a_{e e^{\prime}} M_{e^{\prime}}$ with $a_{e e^{\prime}} \in \mathbb{C}^{*}$ then we obtain the same monomial curve.

Remark 3.2 Delorme's Proposition 9 in [6] implies that every complete intersection semigroup arises as in Theorem 3.1. Already in the three-generator case the minimal tree defining the semigroup need not be unique.

Example If $\operatorname{gcd}(a, b)=\operatorname{gcd}(a, c)=\operatorname{gcd}(c, d)=1$ then the tree

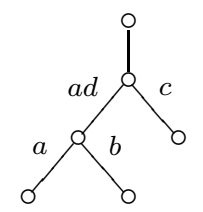

satisfies the semigroup condition and leads to the complete intersection monomial curve

$$
\mathbb{C}\left[z_{1}, z_{2}, z_{3}\right] /\left(z_{1}^{a}-z_{2}^{b}, z_{2}^{d}-z_{3}^{c}\right) \cong \mathbb{C}\left[t^{b c}, t^{a c}, t^{a d}\right] .
$$

Exchanging $a$ with $c$ and $b$ with $d$ gives a different tree for the same semigroup.

Proof of Theorem 3.1 and Scholium The second part of the scholium is an easy induction once the rest is proved, replacing $z_{j} \mapsto t^{\ell w^{\prime} w_{j}}$ for $j>1$ by $z_{j} \mapsto \lambda_{j} t^{\ell_{w^{\prime} w_{j}}}$ for suitable $\lambda_{j} \in \mathbb{C}^{*}$. So we will just prove the theorem and first part of the scholium. 
Let $\Delta_{1}, \ldots, \Delta_{n}$ be the subtrees cut off by the bottom vertex $w_{0}$ of the root edge of $\Delta$ and let $p_{i}$ be the weight on the root edge of $\Delta_{i}$. Write $\Gamma_{i}=\operatorname{sg}\left(\Delta_{i}, w_{0}\right)$, $P=p_{1} \ldots p_{n}$ and $P_{i}=P / p_{i}$. Then

$$
\Gamma=P_{1} \Gamma_{1}+\cdots+P_{n} \Gamma_{n}
$$

the semigroup consisting of all integers of the form $\sum P_{i} \gamma_{i}, \gamma_{i} \in \Gamma_{i}$. Moreover,

$$
\mu\left(\Delta, w^{\prime}\right)=\sum_{i=1}^{n}\left(P_{i} \mu\left(\Delta_{i}, w_{0}\right)-1\right)+(n-1) P+1 .
$$

By Lemma 3.3 below, the desired results now hold for $\Delta$ if they are true for each $\Delta_{i}$. The proof is thus an induction, with the induction start being the case that $\Delta$ consists of only a root edge and $\operatorname{sg}(\Delta)$ is the one-generator semigroup $\mathbb{N}$.

Lemma 3.3 Suppose $\Gamma_{i}$ are semigroups for $i=1, \ldots, n$, and $p_{1}, \ldots, p_{n}$ are pairwise coprime positive integers. Write $P=p_{1} \ldots p_{n}$ and $P_{i}=P / p_{i}$. Let

$$
\Gamma=P_{1} \Gamma_{1}+\cdots+P_{n} \Gamma_{n}
$$

Then:

(1) $2 \delta(\Gamma) \leq \sum_{i=1}^{n} P_{i}\left(2 \delta\left(\Gamma_{i}\right)-1\right)+(n-1) P+1$.

(2) If equality holds in (1) then $p_{i} \in \Gamma_{i}$ for $i=1, \ldots, n$.

(3) $c(\Gamma) \leq \sum_{i=1}^{n} P_{i}\left(c\left(\Gamma_{i}\right)-1\right)+(n-1) P+1$.

(4) If $p_{i} \in \Gamma_{i}$ for $i=1, \ldots, n$ then equality holds in (3).

(5) If each $\Gamma_{i}$ is symmetric then the three statements are equivalent: equality in (11); equality in (3); $p_{i} \in \Gamma_{i}$ for $i=1, \ldots, n$.

(6) Assuming $p_{i} \in \Gamma_{i}$ for each $i$, then $\Gamma$ is symmetric resp. a complete intersection if and only if each $\Gamma_{i}$ is symmetric resp. complete intersection.

(7) If $p_{i} \in \Gamma_{i}$ for each $i$ then one obtains a presentation for $\Gamma$ by adjoining to the disjoint union of presentations for the $\Gamma_{i}$ the $n-1$ relations $w_{1}=$ $\cdots=w_{n}$, where $w_{i}$ is an expression for $p_{i}$ in the presentation of $\Gamma_{i}$.

Proof We shall prove the case $n=2$. The case of general $n$ follows from this case by an easy induction.

To prove (11) we count gaps in $\Gamma$. A gap $\gamma$ of $\Gamma=p_{2} \Gamma_{1}+p_{1} \Gamma_{2}$ is either

(i) one of the $\left(p_{1}-1\right)\left(p_{2}-1\right) / 2$ gaps of $p_{2} \mathbb{N}+p_{1} \mathbb{N}$,

or it is of the form $\gamma=p_{2} \alpha+p_{1} \beta$ for some $\alpha, \beta \in \mathbb{N}$. In this case we will see that either: 
(ii) $\beta$ is the smallest $\beta \in \Gamma_{2}$ in its congruence class $\bmod p_{2}$, and $\alpha \notin \Gamma_{1}$, or

(iii) $0 \leq \alpha<p_{1}$, and $\beta \notin \Gamma_{2}$.

Indeed, if we can express $\gamma$ in the form $\gamma=p_{2} \alpha+p_{1} \beta$ with $\alpha, \beta \in \mathbb{N}$, then we can do so with $0 \leq \alpha<p_{1}$. If this expression does not satisfy condition (iii) then $\beta \in \Gamma_{2}$. In this case decrease $\beta$ by some multiple of $p_{2}$ (maybe zero) to make it the smallest $\beta \in \Gamma_{2}$ in its congruence class $\bmod p_{2}$, and simultaneously increase $\alpha$ by the same multiple of $p_{1}$ to keep $\gamma=p_{2} \alpha+p_{1} \beta$. Since $\gamma$ is a gap of $p_{2} \Gamma_{1}+p_{1} \Gamma_{2}$, we must have $\alpha \notin \Gamma_{1}$, so the expression now satisfies condition (ii).

Now there are exactly $p_{2} \delta\left(\Gamma_{1}\right)$ pairs $(\beta, \alpha)$ satisfying condition (ii) and $p_{1} \delta\left(\Gamma_{2}\right)$ pairs satisfying condition (iii), so there are at most $\left(p_{1}-1\right)\left(p_{2}-1\right) / 2+p_{2} \delta\left(\Gamma_{1}\right)+$ $p_{1} \delta\left(\Gamma_{2}\right)$ gaps of $\Gamma=p_{2} \Gamma_{1}+p_{1} \Gamma_{2}$. This number can be written $\frac{1}{2}\left(p_{2}\left(2 \delta\left(\Gamma_{1}\right)-\right.\right.$ $\left.1)+p_{1}\left(2 \delta\left(\Gamma_{2}\right)-1\right)+p_{1} p_{2}+1\right)$, so part (11) is proven.

This proof shows that we have equality in part (11) if and only if every element $\gamma=p_{2} \alpha+p_{1} \beta$ satisfying condition (ii) or (iii) is a gap of $\Gamma$ and there is no overlap between cases (ii) and (iii). Suppose now $p_{1} \notin \Gamma_{1}$. Then if every $p_{2} \alpha+p_{1} \beta$ satisfying (ii) is a gap of $\Gamma$, there is an overlap: $(\alpha, \beta)=\left(p_{1}, 0\right)$ in condition (ii) shows that $p_{1} p_{2}$ is a gap of $\Gamma$, whence $p_{2} \notin \Gamma_{2}$, so $p_{1} p_{2}$ also has an expression with $(\alpha, \beta)=\left(0, p_{2}\right)$ satisfying condition (iii). Thus $p_{1} \notin \Gamma_{1}$ implies inequality in part (11). Similarly for $p_{2} \notin \Gamma_{2}$, so part (2) is proved.

For statement (3), we show that $i \geq 0$ added to the right hand side of the inequality of part (3) gives an element of $\Gamma$. The sum of the last two terms of

$$
p_{2} c\left(\Gamma_{1}\right)+p_{1} c\left(\Gamma_{2}\right)+\left(p_{1}-1\right)\left(p_{2}-1\right)+i
$$

is in the semigroup generated by $p_{1}$ and $p_{2}$, say $p_{1} \alpha+p_{2} \beta$; so the whole expression equals

$$
p_{2}\left(c\left(\Gamma_{1}\right)+\beta\right)+p_{1}\left(c\left(\Gamma_{2}\right)+\alpha\right),
$$

which by definition of conductors is clearly in $\Gamma$.

For statement (4), suppose $p_{1} \in \Gamma_{1}$ and $p_{2} \in \Gamma_{2}$, but

$p_{2} c\left(\Gamma_{1}\right)+p_{1} c\left(\Gamma_{2}\right)+\left(p_{1}-1\right)\left(p_{2}-1\right)-1=p_{2} \lambda+p_{1} \pi$, for some $\lambda \in \Gamma_{1}, \pi \in \Gamma_{2}$.

Modulo $p_{1}$ this equation says $c\left(\Gamma_{1}\right)-1 \equiv \lambda$, so

$$
c\left(\Gamma_{1}\right)-1=\lambda+p_{1} t, \text { for some integer } t .
$$

Inserting this in the previous equation gives

$$
c\left(\Gamma_{2}\right)-1=\pi+p_{2}(-1-t) .
$$


Since one of $t$ and $-1-t$ is $\geq 0$ and $\lambda, p_{1} \in \Gamma_{1}$ and $\pi, p_{2} \in \Gamma_{2}$, one gets either $c\left(\Gamma_{1}\right)-1 \in \Gamma_{1}$ or $c\left(\Gamma_{2}\right)-1 \in \Gamma_{2}$, a contradiction.

Part (5) is now immediate: (21) and (44) show

$$
\text { (equality in (10) } \Rightarrow\left(p_{1} \in \Gamma_{1} \text { and } p_{2} \in \Gamma_{2}\right) \quad \Rightarrow \quad \text { (equality in (3)), }
$$

and if the $\Gamma_{i}$ are symmetric then $c(\Gamma) \leq 2 \delta(\Gamma)$ and $c\left(\Gamma_{i}\right)=2 \delta\left(\Gamma_{i}\right)$, so equality in (3) implies equality in (11).

Part (6) is proved in 6]. (In this paper we use only that $\Gamma$ is a complete intersection if both $\Gamma_{1}$ and $\Gamma_{2}$ are; this follows from part (17).)

For part (7), let $\Gamma_{1}=\left\langle x_{1}, \ldots, x_{n}: s_{1}, \ldots, s_{k}\right\rangle$ and $\Gamma_{2}=\left\langle y_{1}, \ldots, y_{m}: r_{1}, \ldots, r_{\ell}\right\rangle$ be commutative semigroup presentations of $\Gamma_{1}$ and $\Gamma_{2}$; let $p_{1}=v\left(x_{1}, \ldots, x_{n}\right)$ and $p_{2}=w\left(y_{1}, \ldots, y_{m}\right)$ be expressions for $p_{1}$ and $p_{2}$ in these semigroups. Suppose $p_{2} \gamma_{1}+p_{1} \gamma_{2}=p_{2} \gamma_{1}^{\prime}+p_{1} \gamma_{2}^{\prime}$ equates two elements of $\Gamma=p_{2} \Gamma_{1}+p_{1} \Gamma_{2}$, with $\gamma_{1}, \gamma_{1}^{\prime} \in \Gamma_{1}$ and $\gamma_{2}, \gamma_{2}^{\prime} \in \Gamma_{2}$. Let $\gamma_{1}=g_{1}\left(x_{1}, \ldots, x_{n}\right)$ be an expression for $\gamma_{1} \in \Gamma_{1}$ in terms of the generators (and hence for $p_{2} \gamma_{1}$ in $p_{2} \Gamma_{1}$ ), and similarly $\gamma_{1}^{\prime}=g_{1}^{\prime}\left(x_{1}, \ldots, x_{n}\right), \gamma_{2}=g_{2}\left(y_{1}, \ldots, y_{m}\right), \gamma_{2}^{\prime}=g_{2}^{\prime}\left(y_{1}, \ldots, y_{m}\right)$. Then the relation to be verified in $\Gamma=p_{2} \Gamma_{1}+p_{1} \Gamma_{2}$ is $g_{1}+g_{2}=g_{1}^{\prime}+g_{2}^{\prime}$ (abbreviating $g_{1}\left(x_{1}, \ldots, x_{n}\right)=g_{1}$ etc.), and we must show this follows from the relations of $\Gamma_{1}$ and $\Gamma_{2}$ and the additional relation $v=w$.

With no loss of generality $\gamma_{1} \geq \gamma_{1}^{\prime}$ in $\mathbb{N}$. Then, working in $\mathbb{N}$, we have $p_{2}\left(\gamma_{1}-\right.$ $\left.\gamma_{1}^{\prime}\right)=p_{1}\left(\gamma_{2}^{\prime}-\gamma_{2}\right)$, so $\gamma_{1}-\gamma_{1}^{\prime}=s p_{1}$ and $\gamma_{2}^{\prime}-\gamma_{2}=s p_{2}$ for some $s$ in $\mathbb{N}$. In particular, the equations $g_{1}=s v+g_{1}^{\prime}$ and $g_{2}^{\prime}=s w+g_{2}$ hold in $\Gamma_{1}$ and $\Gamma_{2}$,

so they must follow from the relations of these semigroups. Thus, using the additional relation $v=w$, we deduce $g_{1}+g_{2}=s v+g_{1}^{\prime}+g_{2}=s w+g_{1}^{\prime}+g_{2}=$ $g_{1}^{\prime}+g_{2}^{\prime}$, as desired.

\subsection{Normal form monomials}

The material of this subsection will be needed in Section 5 for the proof of Theorem 2 ,

Suppose now that $\left(\Delta, w^{\prime}\right)$ satisfies the semigroup condition and put $\Gamma=\operatorname{sg}(\Delta)$. We wish to describe a monomial basis for the corresponding complete intersection curve $\mathbb{C}\left[z_{1}, \ldots, z_{m}\right] /$ (relations). That is, we want "normal form" monomials in $z_{1}, \ldots, z_{m}$ so that each $t^{\gamma}$ with $\gamma \in \Gamma$ is the image of exactly one monomial under the map $\mathbb{C}\left[z_{1}, \ldots, z_{m}\right] \rightarrow \mathbb{C}\left[t^{\Gamma}\right]$ given by $z_{j} \mapsto t^{\ell} w^{\prime} w_{j}$. We will do this by systematically trying to eliminate variables with small index. 
We assume that the tree $\Delta$ is drawn so that the indices $i=1, \ldots, m$ of the leaves increase from left to right. For any node $v$ and outward edge $e$ at $v$ let $\Delta_{v e}$ be the subtree below $v$ with root vertex $v$ and root edge $e$.

If $M$ is a monomial, let $M_{v e}$ be the submonomial of $M$ determined by the variables corresponding to leaves of $\Delta_{v e}$. This monomial represents $t^{\alpha} \in \mathbb{C}\left[t^{\mathrm{sg}\left(\Delta_{v e}\right)}\right]$ for some $\alpha$. We will say $M$ is in normal form if for every $v$ and $e$ as above so that $e$ is not the rightmost edge at $v, \alpha-p_{e} \notin \operatorname{sg}\left(\Delta_{v e}\right)$.

If $M$ is not in normal form at some $(v, e)$ then we could replace $M_{v e}$ in $M$ by $M_{v e}^{\prime} M_{e^{\prime}}$ where $e^{\prime}$ is the rightmost edge at $v, M_{v e}^{\prime}$ is a monomial representing $t^{\alpha-p_{e}} \in \mathbb{C}\left[t^{\operatorname{sg}\left(\Delta_{v e}\right)}\right]$ and $M_{e^{\prime}}$ is a monomial representing $t^{p_{e^{\prime}} \in \mathbb{C}}\left[t^{\mathrm{sg}\left(\Delta_{v e^{\prime}}\right)}\right]$.

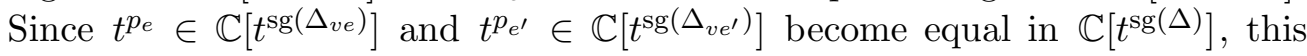
does not change the value of $M$. It is easy to see this process must eventually stop. A simple induction shows that it yields a unique normal form for $M$. Normal form monomials thus provide the desired monomial basis of $\mathbb{C}\left[z_{1}, \ldots, z_{m}\right] /($ relations $)$.

The following example will be important in Section 5 .

Example 5 Let

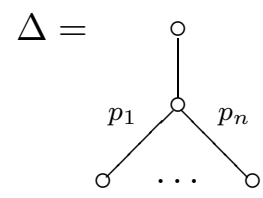

so $\Gamma$ is the semigroup generated by the $P_{i}=P / p_{i}$. The monomial curve

$$
\left(t^{P_{1}}, t^{P_{2}}, \cdots, t^{P_{n}}\right)
$$

is the complete intersection curve singularity defined by the equations

$$
z_{i}^{p_{i}}-z_{n}^{p_{n}}=0, \quad i=1, \cdots, n-1 .
$$

The conductor $c(\Gamma)$ is

$$
P\left(n-1-\sum\left(1 / p_{i}\right)\right)+1
$$

The monomial basis described above is

$$
\left\{z_{1}^{\alpha_{1}} \ldots z_{n}^{\alpha_{n}}: \alpha_{i}<p_{i} \text { for all } i=1, \ldots, n-1\right\}
$$


More generally, applied to a tree of the form

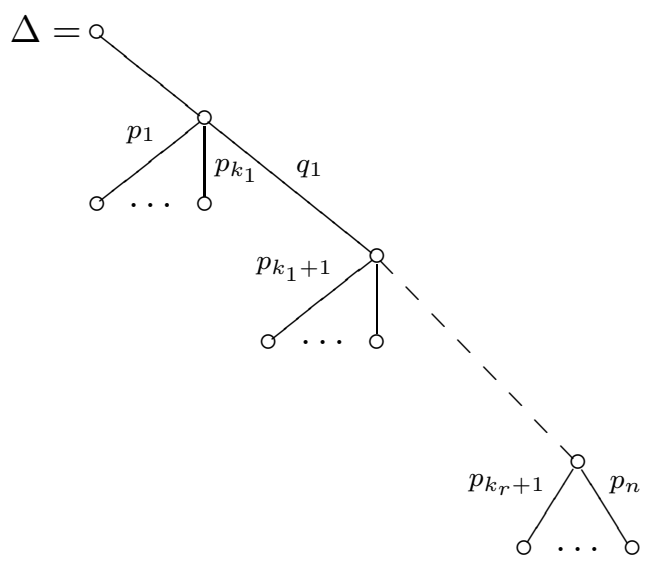

which satisfies the semigroup condition, the above procedure will again give the monomial basis

$$
\left\{z_{1}^{\alpha_{1}} \ldots z_{n}^{\alpha_{n}}: \alpha_{i}<p_{i} \text { for all } i=1, \ldots, n-1\right\} .
$$

(However, with a different ordering of the variables the monomial basis for this example can be considerably more complicated.)

\section{The semigroup condition}

Let $(X, o)$ be a normal surface singularity whose link $\Sigma$ is an integral homology sphere. Each leaf of the splice (or resolution) diagram gives a knot in $\Sigma$, unique up to isotopy. A key point in the proof in [30, that splice diagram equations give integral homology sphere links, is to show that the variable $z_{i}$ associated to a leaf cuts out the corresponding knot in $\Sigma$. In other words, the curve $C_{i}$ given by $z_{i}=0$ is irreducible, and its proper transform $D_{i}$ on the minimal good resolution is smooth and intersects transversely the exceptional curve corresponding to the leaf of the splice diagram. We show that the existence of such functions implies the semigroup condition on the splice diagram.

Theorem 4.1 Let $(X, o)$ be a normal surface singularity whose link $\Sigma$ is an integral homology sphere. Assume that for each of the $t$ leaves $w_{i}$ of the splice diagram $\Delta$ of $\Sigma$, there is a function $z_{i}$ inducing the end knot as above. Then

(1) $\Delta$ satisfies the semigroup condition

(2) $X$ is a complete intersection of embedding dimension $\leq t$ 
(3) $z_{1}, \cdots, z_{t}$ generate the maximal ideal of the local ring of $X$ at $o$, and $X$ is a complete intersection of splice type with respect to these generators.

Proof Let $(Y, E) \rightarrow(X, o)$ be the minimal good resolution, $z=z_{1}$ a function as above, $C \subset X$ the irreducible Cartier divisor defined by $z=0, D \subset Y$ its proper transform, and $E_{1} \subset Y$ the exceptional curve (which intersects $D$ in one point) corresponding to the leaf of the splice diagram.

Let $V$ be the value semigroup of $C$. The orders of vanishing of the functions $z_{2}, \cdots, z_{t}$ at $D \cap E_{1}$ generate a subsemigroup $\Gamma \subset V$ which we can compute from $\Delta$ as follows. For each exceptional curve $E_{i}$, let $a_{i j}$ be the order of vanishing of $z_{j}$ on $E_{i}$, so, as a divisor, $\left(z_{j}\right)=\sum_{i} a_{i j} E_{i}+D_{j}$. The equations $z_{j}^{-1}(0) \cdot E_{k}=0$ imply that $a_{i j}$ is the $i j$-entry of the matrix $\left(-E_{i} \cdot E_{j}\right)^{-1}$; so $a_{i j}=\ell_{i j}$ (see Theorem 9.1). Thus $\Gamma$ is the semigroup generated by $\ell_{1 j}, j \geq 2$.

Theorem 3.1 implies $2 \delta(\Gamma) \leq \mu\left(\Delta, w_{1}\right)$, where $\mu\left(\Delta, w_{1}\right)$ is described there and $\delta(\Gamma)$ denotes the number of gaps of $\Gamma$. But, by Theorem 2.2, $\mu\left(\Delta, w_{1}\right)$ is also equal to the $\mu$-invariant $\mu(C)$ of the curve $C$. Now $\mu(C)=2 \delta(V)$ (since we do not know a priori that the curve is Gorenstein, we must appeal to Buchweitz and Greuel [2] for this). Since the inclusion $\Gamma \subset V$ implies $\delta(V) \leq \delta(\Gamma)$, we conclude that $2 \delta(V)=2 \delta(\Gamma)=\mu\left(\Delta, w_{1}\right)$. Thus $\Gamma=V$, and, by Theorem 3.1 again, $\Gamma=V$ is a complete intersection semigroup. This implies that $C$ is a positive weight deformation of the monomial curve $\mathbb{C}\left[t^{\gamma}: \gamma \in \Gamma\right]$ (eg, Teissier's appendix to [36] or 32]) and in particular is itself a complete intersection (with maximal ideal generated by the images of $\left.z_{2}, \cdots, z_{t}\right)$. It follows that $(X, o)$ is a complete intersection (with maximal ideal generated by $z_{1}, \ldots, z_{n}$ ). Finally, repeating the argument at every leaf gives all the semigroup conditions.

It remains to show that, using the functions $z_{1}, \cdots, z_{t}$ above, we can find splice equations for the singularity. This will proceed as follows: for each node $v$ of valency $\delta=\delta_{v}$, we will write down appropriate monomials in the $z_{i}$ which have the same weight at the node (ie, order of vanishing along the corresponding exceptional curve), and conclude there are $\delta-2$ independent linear dependence relations among these monomials, mod higher weight terms.

Let $E_{v}$ be the exceptional curve corresponding to the node $v$ and let $E_{1}, \ldots, E_{\delta}$ be the exceptional curves with intersect $E_{v}$, corresponding to edges $e_{1}, \ldots, e_{\delta}$ at $v$. Choose a monomial $M_{i}$ of weight $d_{v}$ associated to each edge $e_{i}$ at $v$ (their existence is guaranteed by the semigroup condition). On the exceptional curve $E_{v}$ these monomials all vanish to order $d_{v}$. If we go to an adjacent node 
$v^{\prime}$ of the splice diagram, as in

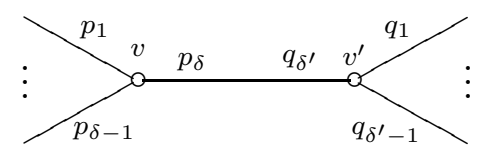

then the order of vanishing of $M_{i}$ is $p_{1} \ldots p_{\delta-1} q_{1} \ldots q_{\delta^{\prime}-1}$ for $i \neq \delta$ and the order of vanishing of $M_{\delta}$ is $p_{\delta} q_{\delta^{\prime}}$. In particular, at the exceptional curve corresponding to $v^{\prime}, M_{\delta}$ vanishes to order $D$ more than the other $M_{i}$ 's, where $D$ is the edge determinant of edge $e_{\delta}$. Now in the (unreduced) maximal splice diagram (see the Appendix; Section 9) we have a node for every exceptional curve and all edge determinants are 1 . Thus we see that on each exceptional curve $E_{i}$ that intersects $E_{v}$, the $M_{j}$ with $j \neq i$ vanish to a common order and $M_{i}$ vanishes to one higher order. Thus, if we fix one of the neighboring exceptional curves, say $E_{\delta}$, then each ratio $M_{i} / M_{\delta}$ for $i \neq \delta$ gives a function on $E_{v}$ that has a pole of order 1 at the point $E_{v} \cap E_{\delta}$, a simple zero at the point of intersection $E_{v} \cap E_{i}$, and no other poles or zeros. It follows that there are $\delta-2$ linearly independent relations among the $M_{i}$ up to higher order at $E_{v}$, as desired.

This gives us a collection of higher weight perturbations of equations of strict splice type and they are the complete intersection description of $(X, o)$ since they give the appropriate complete intersection curves when intersected with $z_{j}=0$.

It is a Riemann-Roch problem to determine if a singularity with homology sphere link has functions $z$ with the properties described above. However, it is not even known if there is any function at all giving an irreducible divisor on $X$; this is certainly not the case for a general hypersurface singularity [13.

We give an application of the above theorem. We will show in Section 8 that if a surface singularity of the form $z^{n}=g(x, y)$ has homology sphere link, then there is a splice type singularity with the same topology (and this singularity is analytically equivalent to one given by an equation of the form $z^{n}=f(x, y)$ ). This leaves open the question whether the original singularity $z^{n}=g(x, y)$ is an equisingular deformation of the strict splice type singularity $z^{n}=f(x, y)$ and is hence of splice type.

Corollary 4.2 Any surface singularity with homology sphere link given by an equation $z^{n}=g(x, y)$ is a splice type singularity. 
Proof We just sketch the proof. If the splice diagram for the plane curve $g(x, y)=0$ is

\begin{tabular}{|c|c|c|}
\hline$p_{1} \quad 1$ & 1 & $p_{k} 1$ \\
\hline$q_{1}$ & $q_{2}$ & $q_{k}$ \\
\hline
\end{tabular}

then it is known (see, eg, 32 ) that curves corresponding to ends of this splice diagram are cut out by polynomials (namely certain "approximate roots" $g_{i}(x, y)$ of $g(x, y))$. It is easy to check that the functions $g_{i}(x, y)$ then cut out curves in the surface $z^{n}=g(x, y)$ corresponding to the ends of its splice diagram

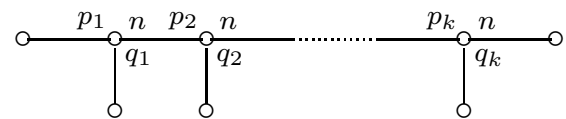

so Theorem 4.1 applies.

\section{$5 \quad$ Geometric genus and Theorem 2}

In this section we will prove Theorem 2 that the Casson Invariant Conjecture holds for a splice type singularity when the nodes of the splice diagram are in a line. We will do this by computing geometric genus $p_{g}$, to prove Version 2 of the Casson Invariant Conjecture in the Introduction. The equivalence of the two versions of the Casson Invariant Conjecture will be proved in Theorem 6.3.

Let $(X, o)$ be a germ of a normal surface singularity, with analytic local ring $\mathcal{O}$. Consider a good resolution $\pi:(Y, E) \rightarrow(X, o)$, ie, the exceptional fiber $E=\bigcup E_{i}$ is a union of smooth curves intersecting transversely, no three through a point. By local duality, one may compute the geometric genus in two ways:

$$
p_{g}(X)=\operatorname{dim} H^{1}\left(\mathcal{O}_{Y}\right)=\operatorname{dim} H^{0}\left(U, K_{U}\right) / H^{0}\left(Y, K_{Y}\right),
$$

where $U=X-\{o\}=Y-E$, and $K$ denotes canonical line bundle (or its sheaf of sections).

If $(X, o)$ is Gorenstein, let $\omega$ be a nowhere-0 holomorphic two-form on $U$. Define the canonical ideal $J$ of $\mathcal{O}$ by

$$
J=\{f \in \mathcal{O}: f \omega \text { is regular on } Y\} .
$$

Then clearly

$$
p_{g}(X)=\operatorname{dim} \mathcal{O} / J .
$$

Let $E_{\alpha}, \alpha=1, \ldots, t$ be those exceptional curves which either have positive genus, or intersect at least three other curves. Let $G$ be the union of the remaining curves (the "strings" in the resolution). The blowing-down $Y \rightarrow Y^{\prime}$ of 
$G$ gives a space with only cyclic quotient singularities (if $Y$ is the minimal good resolution then $Y^{\prime}$ is the "log-canonical resolution"); since these singularities are rational, regular forms in a punctured neighborhood automatically extend regularly on a resolution. Therefore, $f \in J$ if and only if $f \omega$ extends regularly over the $t$ particular curves $E_{\alpha}$. Let $\nu_{\alpha}$ be the valuation on $\mathcal{O}$ given by order of vanishing along $E_{\alpha}$, and let $k_{\alpha}-1$ denote the order of the pole of $\omega$ along that curve. We conclude that

$$
J=\left\{f \in \mathcal{O}: \nu_{\alpha}(f) \geq k_{\alpha}-1, \alpha=1,2, \ldots, t\right\} .
$$

In our case we can improve $k_{\alpha}-1$ to $k_{\alpha}$.

Proposition 5.1 Let $(X, o)$ be the germ of a Gorenstein surface singularity, whose link is a rational homology sphere. Let $(Y, E) \rightarrow(X, o)$ be the minimal good resolution, and let $E_{1}, \cdots, E_{t}$ be the exceptional curves of valency $\geq$ 3. Let $k_{\alpha}$ be the coefficient of $E_{\alpha}$ in the divisor $-(K+E)$, and $\nu_{\alpha}$ the corresponding valuation of the local ring $\mathcal{O}$ of $X$. Then the geometric genus of $X$ is the colength of the ideal

$$
J=\left\{f \in \mathcal{O}: \nu_{\alpha}(f) \geq k_{\alpha}, \alpha=1, \ldots, t\right\} .
$$

Proof By the preceding discussion, the statement to be proved is

$$
H^{0}\left(Y, K_{Y}\right)=H^{0}\left(Y-G, K_{Y}+E\right) .
$$

We will do this in two steps:

$$
H^{0}\left(Y, K_{Y}\right)=H^{0}\left(Y, K_{Y}+E\right)=H^{0}\left(Y-G, K_{Y}+E\right) .
$$

Since the link of $X$ is a $\mathbb{Q}$-homology-sphere, the exceptional curve $E$ is the transverse union of smooth rational curves $E_{i}$, no three through a point, with contractible dual graph. It follows that $h^{1}\left(\mathcal{O}_{E}\right)=0$. (Proof: write $E=E_{1}+F$, where $E_{1}$ is a component of $E$ that meets the rest $F$ of $E$ in a single point; the surjection $\mathcal{O}_{E} \rightarrow \mathcal{O}_{F}$ has kernel $\mathcal{O}_{E_{1}}(-F)=\mathcal{O}(-1)$, so the claim follows by induction on the number of components of $E$.)

Denote $K_{Y} \otimes \mathcal{O}_{E}(E)$ by $K_{E}$ (called the dualizing sheaf in [1, Section II.1). Serre duality implies that, for any line bundle $L$ on $E, H^{1}(E, L)$ is dual to $H^{0}\left(E, L^{*} \otimes K_{E}\right)($ eg, 1], Theorem $\operatorname{II}(6.1))$. Taking $L$ trivial we see $h^{0}\left(K_{E}\right)=0$. The adjunction sequence $0 \rightarrow K_{Y} \rightarrow K_{Y}+E \rightarrow K_{E} \rightarrow 0$ (called "residue sequence" in [1], Section II.1) now gives $0 \rightarrow H^{0}\left(K_{Y}\right) \rightarrow H^{0}\left(K_{Y}+E\right) \rightarrow$ $H^{0}\left(K_{E}\right)=0$, proving the first equality $H^{0}\left(K_{Y}\right)=H^{0}\left(K_{Y}+E\right)$.

The second equality $H^{0}\left(Y, K_{Y}+E\right)=H^{0}\left(Y-G, K_{Y}+E\right)$ holds generally, without the condition on the link. In fact, if $G$ is any union of components of 
$E$ and $L$ any divisor supported on $E$, then it is easy to see that $H^{0}(Y, L)=$ $H^{0}(Y-G, L)$ so long as $L \cdot G_{i} \leq 0$ for each component of $G$ (for a stronger statement see [8]), so we must just show that that $(K+E) \cdot G_{i} \leq 0$ for all $i$. But $G_{i}$ is a smooth rational curve, so $(K+E) \cdot G_{i}$ equals -2 plus the number of intersections of $G_{i}$ with the other curves of $E$. This result is -1 if $G_{i}$ is an end curve of the graph, or 0 otherwise. In either case, the condition is fulfilled, and our result follows.

While the $k_{i}$ are determined from the resolution graph (see Proposition 17.1), in some cases they can be computed directly from the equations defining $\mathcal{O}$.

Proposition 5.2 Let

$$
\mathbb{C}\left[z_{1}, \ldots, z_{s}\right] /\left(f_{1}, \cdots, f_{s-2}\right)
$$

define an isolated complete intersection surface singularity at the origin. For an exceptional curve $E_{1}$ in a resolution, with valuation $\nu=\nu_{1}$, consider the filtration defined by $I_{n}=\{f: \nu(f) \geq n\}$. Assume that the associated graded of this filtration is a complete intersection integral domain, with the $z_{i}$ inducing homogeneous generators, and defined by the $\nu$-leading forms $\overline{f_{j}}, j=1, \cdots, s-$ 2. Then the invariant $k_{1}$ is computed as

$$
k_{1}=\sum_{j=1}^{s-2} \nu\left(\overline{f_{j}}\right)-\sum_{i=1}^{s} \nu\left(z_{i}\right) .
$$

Proof We may interpret

$$
\omega=d z_{1} \wedge \cdots \wedge d z_{s} / d f_{1} \wedge \cdots \wedge d f_{s-2} .
$$

On the associated graded, this gives a two-form of total weight

$$
\Sigma \nu\left(z_{i}\right)-\Sigma \nu\left(\overline{f_{j}}\right) .
$$

In terms of local coordinates in a neighborhood of a general point of $E_{1}$, one finds the order of the pole of $\omega$ is one more than the weight, as desired.

If our singularity is a complete intersection of splice type and $E_{1}$ corresponds to a node $v$ of the splice diagram, then, in the terminology of the preceding section, $\nu\left(z_{i}\right)$ is the $v$-weight of $z_{i}$, so $\nu\left(z_{i}\right)$ is the product of splice diagram weights adjacent to the path from node $v$ to leaf $i$. It is easy to see that the formula of the above proposition is then equivalent to that of Proposition 7.1 
Example 6 The last two propositions give a well-known result for a weighted homogeneous complete intersection: the geometric genus is the sum of the dimensions of the graded pieces of weight less than or equal to $k_{1}$ above. In particular, let $V\left(p_{1}, \ldots, p_{n}\right)$ (with $p_{i}$ pairwise relatively prime) be a Brieskorn complete intersection, defined by

$$
z_{i}^{p_{i}}+a_{i} z_{n-1}^{p_{n-1}}+b_{i} z_{n}^{p_{n}}=0, \quad i=1 \ldots, n-2 .
$$

Let $P=p_{1} \cdots p_{n}, P_{i}=P / p_{i}$. Then

$$
k_{1}=(n-2) P-\Sigma P_{i}=P\left(n-2-\Sigma\left(1 / p_{i}\right)\right) .
$$

Using the monomial basis $z_{1}^{i_{1}} \cdots z_{n}^{i_{n}}$ with $i_{k}<p_{k}, k=1, \cdots, n-2$, one computes

$$
\begin{gathered}
p_{g}\left(V\left(p_{1}, \ldots, p_{n}\right)\right)=\#\left\{\left(i_{1}, i_{2}, \cdots, i_{n}\right) \in\left(\mathbb{Z}_{\geq 0}\right)^{n}: \sum_{k=1}^{n}\left(i_{k}+1\right) / p_{k}<n-2 ;\right. \\
\left.i_{k}<p_{k}, k=1, \cdots, n-2\right\} .
\end{gathered}
$$

To extend this calculation to singularities corresponding to more complicated splice diagrams, we need one monomial basis which works for every filtration defined by a node of the splice diagram.

Suppose we have a complete intersection $(X, p)$ of splice type corresponding to a splice diagram $\Delta$. For convenience of notation we will assume equations of strict splice type (no higher order terms); the identical proofs will handle the general case. Let $\nu$ be the valuation associated to the node $v$ of $\Delta$ (see Theorem 2.1). Let the edges around $v$ be $e_{1}, \ldots, e_{n}$ with weights $d_{v e_{i}}=p_{i}, i=1, \ldots, n$ at $v$. For each node $v^{\prime}$ of $\Delta$ the equations have the form $\sum a_{e^{\prime}} M_{v^{\prime} e^{\prime}}=0$, sum over the edges $e^{\prime}$ at $v^{\prime}$, where $M_{v^{\prime} e^{\prime}}$ is an admissible monomial at $v^{\prime}$ and $a_{e^{\prime}} \in \mathbb{C}$. If $v^{\prime} \neq v$ and $e^{\prime}$ is the edge on the path from $v^{\prime}$ to $v$ we will call $M_{v^{\prime} e^{\prime}}$ a near monomial at $v^{\prime}$ for $v$. Thus there is one near monomial for $v$ associated to each node other than $v$.

Theorem 5.3 The associated graded ring $R$ of $(X, p)$ with respect to the filtration associated to $\nu$ is a reduced and irreducible complete intersection, defined by the same equations as $(X, p)$ but with the coefficients of all near monomials for $v$ set to zero (so only the equations associated to the node $v$ remain unchanged). Its normalization is the Brieskorn complete intersection $V\left(p_{1}, \cdots, p_{n}\right)$.

We will need a specific basis of the graded ring $R$. 
Proposition 5.4 Choose an edge $e$ at $v$ and picture the edge $e$ as horizontal, with $v$ on the left. Cut the edge $e$ at its midpoint and use this midpoint as root of the resulting trees $\Delta_{e}^{L}$ on the left and $\Delta_{e}^{R}$ on the right (so these are rooted trees and $\Delta_{e}^{L}$ contains $v$ ). Let $\mathcal{M}_{e}^{L}$ and $\mathcal{M}_{e}^{R}$ be monomial bases for the monomial curves of $\Delta_{e}^{L}$ and $\Delta_{e}^{R}$, constructed as in subsection [3.1. Then the set of monomials $\mathcal{M}_{e}^{L} \mathcal{M}_{e}^{R}=\left\{M_{1} M_{2}: M_{1} \in \mathcal{M}_{e}^{L}, M_{2} \in \mathcal{M}_{e}^{R}\right\}$ forms a $\mathbb{C}$-basis of the associated graded ring $R$. The integer $k_{v}$ is given by

$$
k_{v}=d_{v e}\left(C^{L}-1\right)+\frac{d_{v}}{d_{v e}}\left(C^{R}-1\right) \quad\left(=p_{j}\left(C^{L}-1\right)+P_{j}\left(C^{R}-1\right) \text { if } e=e_{j}\right)
$$

where $C^{L}$ and $C^{R}$ are the conductors of the semigroups $\operatorname{sg}\left(\Delta_{e}^{L}\right)$ and $\operatorname{sg}\left(\Delta_{e}^{R}\right)$.

Proof of Theorem 5.3 and Proposition 5.4 Let $\nu^{\prime}$ be the valuation associated to $v^{\prime}$. If $z_{w}$ is the variable associated to a leaf $w$ then one checks easily that

$$
\frac{\nu\left(z_{w}\right)}{\nu^{\prime}\left(z_{w}\right)}=\frac{\ell_{v^{\prime} v}}{d_{v^{\prime}}} D_{e_{1}^{\prime}} \ldots D_{e_{k}^{\prime}}
$$

where:

- $\ell_{v^{\prime} v}$ is, as usual, the product of weights adjacent to the path from $v^{\prime}$ to $v$

- $d_{v^{\prime}}$ is the product of edge weights at $v^{\prime}$;

- $e_{1}^{\prime}, \ldots, e_{k}^{\prime}$ are the edges that are on the path from $v^{\prime}$ to $v$ but not on the path from $w$ to $v$;

- for any edge $e, D_{e}$ is the product of the edge weights on $e$ divided by the product of edge weights directly adjacent to $e$ (so $D_{e}>1$ by the edge determinant condition).

Thus $\nu\left(z_{w}\right) / \nu^{\prime}\left(z_{w}\right)$ takes its minimum value (namely $\ell_{v^{\prime} v} / d_{v^{\prime}}$ ) if and only if $w$ is beyond $v^{\prime}$ from the point of view of $v$. It follows that the admissible monomials at $v^{\prime}$ all have the same $\nu$-weight except for the near monomial for $v$, which has higher $\nu$-weight. Hence, the ideal defining the associated graded ring $R$ contains polynomials obtained from our equations by setting coefficients of near monomials equal to zero. We will show these generate an ideal whose quotient is an integral domain of dimension 2, hence yield the full associated graded.

For convenience of notation we will take $e=e_{n}$ for this proof. We may assume (see Section 2) that the equations associated to the node $v$ are

$$
M_{e_{i}}+a_{i} M_{e_{n-1}}+b_{i} M_{e_{n}}=0, \quad i=1, \ldots, n-2 .
$$


For each $j=1, \ldots, n$, let $\Delta_{j}$ be the tree cut off away from $v$ at the midpoint of $e_{j}$ (so $\Delta_{n}=\Delta_{e}^{R}$ ). By the Scholium to Theorem 3.1, the equations for the associated graded $R$ that correspond to nodes in $\Delta_{j}$ give a complete intersection description of the monomial curve $\mathbb{C}\left[X_{j}^{\mathrm{sg}\left(\Delta_{j}\right)}\right]$. Let $\phi_{j}: \mathbb{C}\left[z_{w}, w\right.$ a leaf of $\left.\Delta_{j}\right] \rightarrow$ $\mathbb{C}\left[X_{j}^{\mathrm{sg}\left(\Delta_{j}\right)}\right]$ be the corresponding homomorphism. Then $\phi_{j}\left(M_{e_{j}}\right)=c_{j} X^{p_{j}}$ for some $c_{j} \in \mathbb{C}^{*}$. Together these homomorphisms $\phi_{j}$ give a homomorphism $\phi$ of $R$ to the Brieskorn complete intersection defined by the equations

$$
c_{i} X_{i}^{p_{i}}+c_{n-1} a_{i} X_{n-1}^{p_{n-1}}+c_{n} b_{i} X_{n}^{p_{n}}=0, \quad i=1, \ldots, n-2 .
$$

Let $z_{1}, \ldots, z_{k}$ be the variables corresponding to nodes of $\Delta$ in $\Delta_{e}^{L}$ and let $z_{k+1}, \ldots, z_{N}$ be the remaining variables, corresponding to nodes in $\Delta_{e}^{R}$. The graded equations corresponding to nodes in $\Delta_{e}^{L}$ are equations for the complete intersection curve defined by $\Delta_{e}^{L}$ except for additional terms $b_{i} M_{e_{n}}$ (in the equations corresponding to node $v$ ). The procedure of subsection 3.1 to put a monomial in normal form will therefore change a monomial $M$ in the variables $z_{1}, \ldots, z_{k}$ into a linear combination of monomials of the form $M^{\prime} M_{e_{n}}^{\alpha}, \alpha \geq$ 0 , with $M^{\prime} \in \mathcal{M}_{e}^{L}$. Thus, given any monomial in $z_{1}, \ldots, z_{N}$, we first apply the graded equations corresponding to nodes in $\Delta_{e}^{L}$ to put anything involving $z_{1}, \ldots, z_{k}$ in $\mathcal{M}_{e}^{L}$-normal form (at the expense of adding factors $M_{e_{n}}$ ), and then apply the graded equations corresponding to nodes in $\Delta_{e}^{R}$ to put anything involving $z_{k+1}, \ldots, z_{N}$ into $\mathcal{M}_{e}^{R}$-normal form. It follows that the set $\mathcal{M}_{e}^{L} \mathcal{M}_{e}^{R}$ is a $\mathbb{C}$-spanning set for the graded ring $R$. On the other hand, one can check that the set

$$
\left\{\phi\left(M_{1} M_{2}\right): M_{1} \in \mathcal{M}_{e}^{L}, M_{2} \in \mathcal{M}_{e}^{R}\right\} \subset \mathbb{C}\left[X_{1}, \ldots, X_{n}\right] /(\text { relations (3) })
$$

is linearly independent (we will not give a detailed proof of this, since it is immediate in the case below to which we apply this proposition). Hence $\mathcal{M}_{e}^{L} \mathcal{M}_{e}^{R}$ is a monomial basis for $R$. Moreover, since $\phi$ is birational, $\phi$ is the normalization of $R$. Finally, the calculation of $k_{v}$ is straightforward, using either Proposition 5.2 or Proposition 7.1 .

Note that the monomial basis given by the above proposition depends on the choice of edge and also on the ordering of the variables. Although the proposition gives the same monomial basis for the valuations corresponding to the two ends of the edge, if we take a different node we will have to take a different edge and will in general get a different monomial basis. However, to apply this proposition to compute the geometric genus of $(X, p)$ we shall need the same monomial basis for all the valuations. This turns out to be possible for splice 
diagrams of the type

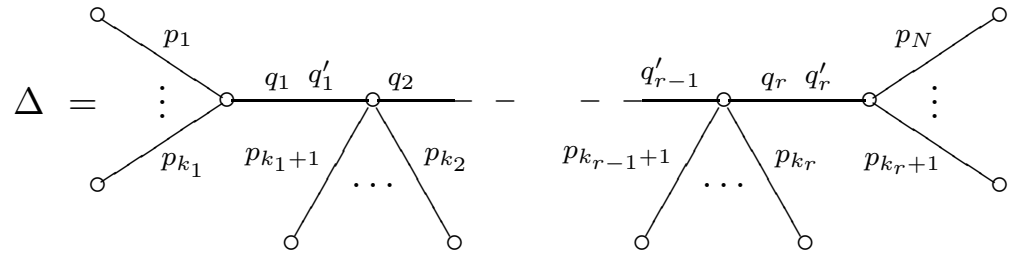

We number the nodes and edges of this diagram $v_{0}, \ldots, v_{r}$ and $e_{1}, \ldots, e_{r}$ from left to right. The valuation for node $v_{i}$ will be denoted $\nu_{i}$.

For the edge $e=e_{i}$ joining nodes $v_{i-1}$ and $v_{i}$ we divide the variables $z_{1}, \ldots, z_{N}$ into two groups, ordered as follows:

$$
\begin{gathered}
z_{k_{i}}, z_{k_{i}-1}, \ldots, z_{1}, \\
z_{k_{i}+1}, z_{k_{i}+2}, \ldots, z_{N}
\end{gathered}
$$

We apply the above proposition for this particular edge $e$. Example 5 gives the monomial bases

$$
\begin{aligned}
& \mathcal{M}_{e}^{L}=\left\{z_{1}^{\alpha_{1}} \ldots z_{k_{i}}^{\alpha_{k_{i}}}: 0 \leq \alpha_{i}<p_{i} \text { for } i=2, \ldots, k_{i}\right\} \\
& \mathcal{M}_{e}^{R}=\left\{z_{k_{i}+1}^{\alpha_{k_{i}+1}} \ldots z_{N}^{\alpha_{N}}: 0 \leq \alpha_{i}<p_{i} \text { for } i=k_{i}+1, \ldots, N-1\right\}
\end{aligned}
$$

for the two semigroups in question, so we get:

Lemma 5.5 For each valuation $\nu_{i}$ of the above $\Delta$,

$$
\mathcal{M}:=\left\{z_{1}^{\alpha_{1}} \ldots z_{N}^{\alpha_{N}}: 0 \leq \alpha_{i}<p_{i} \text { for } i=2, \ldots, N-1\right\}
$$

is a monomial basis for the associated graded ring $R$.

We continue to consider the edge $e=e_{i}$ of $\Delta$ with left end node $v=v_{i-1}$. We can consider $\Delta_{e}^{L}$ and $\Delta_{e}^{R}$ also as splice diagrams, and then $\Delta$ is the result of splicing them at their root leaves.

Theorem 5.6 The geometric genus $p_{g}(\Delta)$ of the splice type singularity determined by $\Delta$ is given inductively by

$$
(1 / 4) C^{L} C^{R}+p_{g}\left(\Delta_{e}^{L}\right)+p_{g}\left(\Delta_{e}^{R}\right)
$$

where $C^{L}, C^{R}$ are the conductors of the semigroups $\operatorname{sg}\left(\Delta_{e}^{L}, w^{\prime}\right)$ and $\operatorname{sg}\left(\Delta_{e}^{R}, w^{\prime}\right)$

The following is a corollary of this and of Theorem 6.3 in the next section.

Corollary 5.7 The Casson Invariant Conjecture holds for the splice type singularity determined by the above splice diagram $\Delta$ 
Proof of Corollary The above theorem reduces this to an induction. The induction step is provided by Theorem 6.3] since $C^{L}$ and $C^{R}$ are the Milnor numbers of the knots corresponding to the root leaves of $\Delta_{e}^{L}$ and $\Delta_{e}^{R}$ (Theorem 2.2).

Proof of Theorem 5.6 The canonical ideal of the singularity $(X, p)$ consists of those $f$ for which $\nu_{j}(f) \geq k_{v_{j}}$ for $j=0, \ldots, r$. Using the linearly independent monomials of the above lemma, the geometric genus thus equals the number of elements $M$ of

$$
\mathcal{M}=\left\{z_{1}^{\alpha_{1}} \ldots z_{N}^{\alpha_{N}}: 0 \leq \alpha_{i}<p_{i} \text { for } i=2, \ldots, N-1\right\}
$$

satisfying

$$
\nu_{i}(M)<k_{v_{i}} \quad \text { for some } i=0, \ldots, r .
$$

We will call this condition "condition $K\left(v_{i}\right)$." So we want to count the $M \in \mathcal{M}$ for which condition $K(v)$ holds for some node $v$.

Let $e=e_{i}$. For a monomial $M=z_{1}^{\alpha_{1}} \ldots z_{N}^{\alpha_{N}}$, write $M=M_{L} M_{R}$ with $M_{L}=$ $z_{1}^{\alpha_{1}} \ldots m_{k_{i}}^{\alpha_{k_{i}}}$ and $M_{R}=z_{k_{i}+1}^{\alpha_{k_{i}+1}} \ldots z_{N}^{\alpha_{N}}$. The monomial $M$ is in $\mathcal{M}$ if and only if $M_{L}$ and $M_{R}$ are normal form monomials for the semigroups $\operatorname{sg}\left(\Delta_{e}^{L}\right)$ and $\operatorname{sg}\left(\Delta_{e}^{R}\right)$.

Denote the nodes at the left and right end of $e=e_{i}$ by $v=v_{i-1}$ and $v^{\prime}=v_{i}$ and the associated valuations by $\nu=\nu_{i-1}$ and $\nu^{\prime}=\nu_{i}$. Denote

$$
\ell_{e}\left(M_{L}\right):=\sum_{j=1}^{k_{i}} \alpha_{j} \ell_{w^{\prime} w_{j}}, \quad \ell_{e}\left(M_{R}\right):=\sum_{j=k_{i}+1}^{N} \alpha_{j} \ell_{w^{\prime} w_{j}}
$$

where $w^{\prime}$ is the root vertex of $\Delta_{e}^{L}$ or $\Delta_{e}^{R}$ and $\ell_{w^{\prime} w_{j}}$ is computed in $\Delta_{e}^{L}$ or $\Delta_{e}^{R}$. (Thus $\ell_{e}\left(M_{L}\right)$ and $\ell_{e}\left(M_{R}\right)$ are the values in the $\operatorname{semigroups} \operatorname{sg}\left(\Delta_{e}^{L}\right)$ and $\operatorname{sg}\left(\Delta_{e}^{R}\right)$ corresponding to the monomials $M_{L}$ and $\left.M_{R}.\right)$ Then

$$
\nu(M)=d_{v e} \ell_{e}\left(M_{L}\right)+\left(d_{v} / d_{v e}\right) \ell_{e}\left(M_{R}\right) .
$$

By Proposition 5.4 condition $K\left(v_{i-1}\right)$ can thus be written

$$
d_{v e}\left(\ell_{e}\left(M_{L}\right)-C^{L}+1\right)+\left(d_{v} / d_{v e}\right)\left(\ell_{e}\left(M_{R}\right)-C^{R}+1\right)<0
$$

By symmetry, condition $K\left(v_{i}\right)$ can be written

$$
\left(d_{v^{\prime}} / d_{v^{\prime} e}\right)\left(\ell_{e}\left(M_{L}\right)-C^{L}+1\right)+d_{v^{\prime} e}\left(\ell_{e}\left(M_{R}\right)-C^{R}+1\right)<0 .
$$

Denote

$$
X_{i}:=\ell_{e}\left(M_{L}\right)-C^{L}+1 \quad Y_{i}:=\ell_{e}\left(M_{R}\right)-C^{R}+1,
$$


so (5) and (6) can be written

$$
\begin{array}{rr}
K\left(v_{i-1}\right): & d_{v e} X_{i}+\left(d_{v} / d_{v e}\right) Y_{i}<0, \\
K\left(v_{i}\right): & \left(d_{v^{\prime}} / d_{v^{\prime} e}\right) X_{i}+d_{v^{\prime} e} Y_{i}<0 .
\end{array}
$$

Note that $X_{i} \neq 0$ since $\ell_{e}\left(M_{L}\right)$ is in the semigroup $\operatorname{sg}\left(\Delta_{e}^{L}\right)$ with conductor $C^{L}$. Similarly $Y_{i} \neq 0$. We will count the monomials $M \in \mathcal{M}$ that satisfy condition $K(v)$ for some node $v$ by subdividing into the following cases.

(1) $X_{i}<0$ and $Y_{i}<0$ (so $K\left(v_{i-1}\right)$ and $K\left(v_{i}\right)$ hold),

(2) $Y_{i}>0$ and $K\left(v_{j}\right)$ holds for some $j \leq i-1$,

(3) $X_{i}>0$ and $K\left(v_{j}\right)$ holds for some $j \geq i$,

(4) $Y_{i}>0$ and $K\left(v_{j}\right)$ holds for some $j \geq i$ and fails for all $j \leq i-1$.

(5) $X_{i}>0$ and $K\left(v_{j}\right)$ holds for some $j \leq i-1$ and fails for all $j \geq i$,

These cases cover all possibilities. We shall show that cases (4) and (5) are empty and that Cases (1), (2), (3) are mutually exclusive and lead to the three terms on the right in the theorem.

(1) The number of monomials $M_{L}$ in normal form with $\ell_{e}\left(M_{L}\right)<C^{L}-1$ is the number of elements bounded by $C^{L}$ in the semigroup $\operatorname{sg}\left(\Delta_{e}^{L}\right)$. This is exactly $C^{L} / 2$. Similarly for $M_{R}$, so the set of $M \in \mathcal{M}$ with both $\ell_{e}\left(M_{L}\right)-C^{L}+1<0$ and $\ell_{e}\left(M_{R}\right)-C^{R}+1<0$ contributes the $(1 / 4) C^{L} C^{R}$ of the theorem.

(2) The inequality $\ell_{e}\left(M_{R}\right)-C^{R}+1>0$ says $\ell_{e}\left(M_{R}\right) \geq C^{R}$, so there exists a unique monomial $M_{R}$ in normal form with such a value of $\ell_{e}\left(M_{R}\right)$. That is, if we put $\alpha=\ell_{e}\left(M_{R}\right)-C^{R}$ then there is no constraint on $\alpha \geq 0$ for a corresponding $M_{R}$ to exist. Consider the monomials $M_{L} M_{R}$ and $M_{L} z^{\alpha}$, which are normal form monomials for the splice diagrams $\Delta$ and $\Delta_{e}^{L}$ respectively. A simple calculation, which we omit, shows that $M_{L} M_{R}$ satisfies condition $K\left(v_{j}\right)$ for $\Delta$ with $j \leq i-1$ if and only if $M_{L} z^{\alpha}$ satisfies $K\left(v_{j}\right)$ for $\Delta_{e}^{L}$. Thus the monomials $M=M_{L} M_{R}$ satisfying (2) are in one-one correspondence with the monomials that count $p_{g}\left(\Delta_{e}^{L}\right)$.

(3) By symmetry, these monomials count $p_{g}\left(\Delta_{e}^{R}\right)$.

(4) One calculates that

$$
Y_{i}=\left(d_{v_{i}} / q_{i}^{\prime} q_{i+1}\right) Y_{i+1}+\left(d_{v_{i}} / q_{i}\right) \sum_{j=k_{i}+1}^{k_{i+1}} \frac{1}{p_{j}}\left(\alpha_{j}+1-p_{j}\right)
$$

(we are using the explicit weights $d_{v_{i} e}=q_{i}^{\prime}$ etc. from the picture of $\Delta$ ). Since $\alpha_{j}<p_{j}$ for $j=k_{i}+1, \ldots, k_{i+1}$, the sum on the right is non-positive so $Y_{i}>0$ 
implies $Y_{i+1}>0$. Thus, if we are in case (4) we can, by increasing $i$ if necessary, assume that $Y_{i}>0$ and $K\left(v_{j}\right)$ holds for $j=i$ and fails for $j=i-1$. By (7) we then have

$$
d_{v} / d_{v e}^{2}>-X_{i} / Y_{i}, \quad d_{v^{\prime} e}^{2} / d_{v}^{\prime}<-X_{i} / Y_{i} .
$$

Thus $d_{v} / d_{v e}^{2}>d_{v^{\prime} e}^{2} / d_{v}^{\prime}$, whence $d_{v} d_{v^{\prime}} /\left(d_{v e} d_{v^{\prime} e}\right)>d_{v e} d_{v^{\prime} e}$, contradicting the edge determinant condition. Thus case (4) cannot happen, and by symmetry the same holds for case (5).

It remains to show that cases (2) and (3) are mutually exclusive (Case (1) is clearly disjoint from (2) and (3)). But if both (2) and (3) hold then $K\left(v_{i}\right)$ must fail. Since $K\left(v_{j}\right)$ holds for some $j>i$ and $Y_{i}>0$, the same argument as in (4) leads to a contradiction.

\section{Milnor fibers}

Suppose $\Sigma$ is the link of an isolated singularity at 0 of a complete intersection surface $X=f^{-1}(0)$, where $f$ is a map $f=\left(f_{1}, \ldots, f_{n-2}\right):\left(\mathbb{C}^{n}, 0\right) \rightarrow\left(\mathbb{C}^{n-2}, 0\right)$. The Milnor fiber is the manifold $F:=f^{-1}(\delta) \cap B(\epsilon)$ where $B(\epsilon)$ is a sufficiently small ball about 0 and $\delta$ is a general point of $\mathbb{C}^{n-2}$ very close to the origin. It is a smooth simply-connected piece of complex surface with boundary $\Sigma$; it has a symmetric intersection pairing on the second homology group, whose rank $b_{2}(F)$ is usually denoted by $\mu$. The Casson Invariant Conjecture says that when $\Sigma$ is a homology sphere, $\operatorname{sign}(F)$ should equal $8 \lambda(\Sigma)$, where $\lambda(\Sigma)$ is the Casson invariant.

The Casson invariant of $\Sigma$ is not hard to compute, and the hurdle in confirming this conjecture for any particular example is to understand $F$ well enough to compute $\operatorname{sign}(F)$. This has been done for Brieskorn complete intersections. Thus, the conjecture could be verified in this case - a one-node splice diagram (see [26], which also proves a few other cases).

Now suppose the equations $f_{i}\left(z_{1}, \ldots, z_{n}\right)=0, i=1, \ldots, n-2$, are of splice type as above, corresponding to a splice diagram $\Delta$. Thus the curve $z_{j}=0$ cuts out in $\Sigma$ the knot $K_{j}$ corresponding to the $j$-th leaf of $\Delta$. The link $\left(\Sigma, K_{j}\right)$ is a fibered link whose fiber $G_{j}$ can also be seen as the Milnor fiber of the singularity at 0 of the complete intersection curve $\left(f_{1}, \ldots, f_{n-2}, z_{j}\right)^{-1}(0)$. The topology of this fiber and its embedding in $\Sigma$ can be described by gluing together Milnor fibers of appropriate links in the splice components of $\Sigma$ (see [7]). 
We shall describe a conjectural iterative description of $F$ in terms of the Milnor fibers of simpler complete intersection surface singularities and fibers $G_{j}$ as above lying in their boundaries.

Thus consider $\Sigma$ as the splice $\Sigma=\Sigma_{1} \frac{K_{1} \quad K_{2}}{2} \Sigma_{2}$ of two homology spheres determined by cutting $\Delta$ at an edge to form two rooted diagrams. It is easy to see that these two diagrams $\Delta_{1}$ and $\Delta_{2}$ also satisfy the semigroup condition so $\Sigma_{1}$ and $\Sigma_{2}$ are both complete intersection singularity links given by equations of splice type. They thus have Milnor fibers, which we shall call $F_{1}$ and $F_{2}$, with $\partial F_{i}=\Sigma_{i}$.

Let $G_{1} \subset \Sigma_{1}$ be the fiber for the knot $\left(\Sigma_{1}, K_{1}\right)$. This is the Milnor fiber described in the paragraph before Theorem 2.2, so it is topologically determined by the rooted diagram $\Delta_{1}$ and $b_{1}\left(G_{1}\right)$ is computed as in that theorem.

We may push the embedding $G_{1} \rightarrow F_{1}$ inside $F_{1}$ by a normal vector-field to obtain a proper embedding $G_{1} \rightarrow F_{1}$ (that is, an embedding with $\partial G_{1}=$ $G_{1} \cap \partial F_{1}$, transverse intersection) and then extend to an embedding $G_{1} \times D^{2} \rightarrow$ $F_{1}$ of a tubular neighborhood of $G_{1}$. We similarly construct an embedding $D^{2} \times G_{2} \rightarrow F_{2}$.

Denote

$$
F_{1}^{o}:=F_{1}-\left(G_{1} \times \stackrel{\circ}{D}^{2}\right), \quad F_{2}^{o}:=F_{2}-\left(\stackrel{\circ}{D}^{2} \times G_{i}\right),
$$

so $\partial F_{1}^{o}$ is the union of $G_{1} \times S^{1}$ and the exterior (complement of an open tubular neighborhood) of the knot $K_{1} \subset \Sigma_{1}$, and similarly for $\partial F_{2}^{o}$.

Conjecture 2 (Milnor Fiber Conjecture) $F$ is homeomorphic to the result $\bar{F}$ of pasting:

$$
\bar{F}:=F_{1}^{o} \cup_{G_{1} \times S^{1}}\left(G_{1} \times G_{2}\right) \cup_{S^{1} \times G_{2}} F_{2}^{o},
$$

where we identify $G_{1} \times S^{1}$ with $G_{1} \times \partial G_{2}$ and $S^{1} \times G_{2}$ with $\partial G_{1} \times G_{2}$.

By Milnor [18] and Hamm [9],F, $F_{1}, F_{2}$ are simply connected 4-manifolds which are homotopy equivalent to 2 -complexes and thus have reduced homology only in dimension 2 . We show that $\bar{F}$ has the nice properties we would like $F$ to have.

Theorem 6.1 $\partial \bar{F}=\Sigma$ and $\bar{F}$ is simply connected and homotopy equivalent to a 2-complex. Moreover,

$$
\begin{aligned}
H_{2}(\bar{F}) & \cong H_{2}\left(G_{1} \times G_{2}\right) \oplus H_{2}\left(F_{1}\right) \oplus H_{2}\left(F_{2}\right) \\
& =\left(H_{1}\left(G_{1}\right) \otimes H_{1}\left(G_{2}\right)\right) \oplus H_{2}\left(F_{1}\right) \oplus H_{2}\left(F_{2}\right) .
\end{aligned}
$$


with maps induced by inclusions, so

$$
\operatorname{sign}(\bar{F})=\operatorname{sign}\left(F_{1}\right)+\operatorname{sign}\left(F_{2}\right) .
$$

Corollary 6.2 The Milnor Fiber Conjecture (Conjecture2) implies the Casson Invariant Conjecture for complete intersection singularities of splice type.

Proof The theorem and Conjecture 2 imply that signature of Milnor fiber is additive under splicing. The Casson invariant is additive for splicing. The Casson Invariant Conjecture is known for Brieskorn complete intersections (the one-node case).

Proof of Theorem 6.1 The fact that $\partial \bar{F}=\Sigma$ is immediate from the construction. For the rest of this proof it is convenient to have a different description of $\bar{F}$.

Consider $G_{i}$ embedded in $\Sigma_{i}$ and let $N_{i} \subset \Sigma_{i}=\partial F_{i}$ be a tubular neighborhood of $G_{i}$ in $\Sigma_{i}$, so $N_{i} \cong G_{i} \times I$. Note that $\partial\left(G_{1} \times G_{2}\right)=\left(G_{1} \times K_{2}\right) \cup\left(K_{1} \times G_{2}\right)$, so we can also embed $N_{1}$ in $\partial\left(G_{1} \times G_{2}\right)$ as $G_{1} \times I \subset G_{1} \times K_{2}$, and similarly for $N_{2}$. We claim:

$$
\bar{F} \cong F_{1} \cup_{N_{1}}\left(G_{1} \times G_{2}\right) \cup_{N_{2}} F_{2} .
$$

Indeed, to turn our previous description of $\bar{F}$ into this one, connect the proper embedding $G_{i} \subset F_{i}$ to the embedding $G_{i} \subset \partial F_{i}$ by a "strip" $G_{i} \times I$ and remove a tubular neighborhood of this strip from $F_{i}^{o}$ and glue it onto $G_{1} \times G_{2}$ instead. The result of removing it from $F_{i}^{o}$ is something homeomorphic to $F_{i}$, while, when glued to $G_{1} \times G_{2}$ it is just a collar on part of the boundary and does not change the homeomorphism type of $G_{1} \times G_{2}$.

Consider, therefore, $\bar{F}$ as in equation (8). By shrinking slightly the regions $N_{i}$ along which the $F_{i}$ are glued to $G_{1} \times G_{2}$ we can make them disjoint in $\partial\left(G_{1} \times G_{2}\right)$ without changing the homotopy type (or even homeomorphism type) of $F_{1} \cup_{N_{1}}\left(G_{1} \times G_{2}\right) \cup_{N_{2}} F_{2}$. Then $\left(G_{1} \times G_{2}\right) \cap\left(F_{1} \cup F_{2}\right)$ consists of the disjoint union of $N_{1}$ and $N_{2}$. The Meyer-Vietoris sequence for the decomposition $\left(G_{1} \times\right.$ $\left.G_{2}\right) \cup\left(F_{1} \cup F_{2}\right)$ then easily yields that the inclusions induce an isomorphism

$$
H_{2}(\bar{F}) \cong H_{2}\left(G_{1} \times G_{2}\right) \oplus H_{2}\left(F_{1}\right) \oplus H_{2}\left(F_{2}\right)
$$

as desired.

The fact that $\bar{F}$ is simply connected is an easy application of the Van Kampen theorem. The fact that $\bar{F}$ is homotopy equivalent to a 2 -complex can be seen by replacing $G_{1}$ and $G_{2}$ by one-dimensional spines $S_{1}$ and $S_{2}$ say, replacing $F_{1}$ and $F_{2}$ by 2 -dimensional spines $T_{1}$ and $T_{2}$, and then gluing $S_{1} \times S_{2}$ to $T_{1}$ and $T_{2}$ by means of mapping cylinders of appropriate maps $S_{i} \rightarrow T_{i}$. 
Recall that the geometric genus $p_{g}(X, o)$ of a singularity is $\operatorname{dim} H^{1}(Y, \mathcal{O})$, where $Y \rightarrow X$ denotes a resolution of the singularity. In general, it is not topologically determined by the link of $X$, but the Casson Invariant Conjecture says that it should be for complete intersection singularities with homology sphere links. The following theorem says what the Casson Invariant Conjecture implies about the behavior of various invariants under splicing. Item (3) of this theorem provided part of the motivation for the above construction of $\bar{F}$ for the Milnor Fiber Conjecture.

Theorem 6.3 Let $X$ be a complete intersection with homology sphere link, with Milnor fiber $F$; and suppose its link is spliced from links of two singularities $X_{1}, X_{2}$, with Milnor fibers $F_{1}, F_{2}$. Assume the Casson Invariant Conjecture for $X_{1}$ and $X_{2}$. Then the following statements are equivalent:

(1) The Casson Invariant Conjecture holds for $X$.

(2) We have $\operatorname{sign}(F)=\operatorname{sign}\left(F_{1}\right)+\operatorname{sign}\left(F_{2}\right)$.

(3) With $G_{1}, G_{2}$ as above, we have $b_{2}(F)=b_{2}\left(F_{1}\right)+b_{2}\left(F_{2}\right)+b_{1}\left(G_{1}\right) b_{1}\left(G_{2}\right)$, where $b_{i}$ is Betti number.

(4) The geometric genus satisfies $p_{g}(X)=p_{g}\left(X_{1}\right)+p_{g}\left(X_{2}\right)+\frac{1}{4} b_{1}\left(G_{1}\right) b_{1}\left(G_{2}\right)$.

Moreover, these invariants of $X$ are then topologically determined by the link.

Proof The equivalence of (1) and (2) has already been discussed, so we prove the equivalence of (2), (3), and (4).

Formulas of H. Laufer and A. Durfee imply that the geometric genus of X, and the signature and second Betti number $\mu$ of the Milnor fiber, are explicitly related by topological invariants of the link (see, eg, 34.) Let $Y \rightarrow X$ denote a good resolution, and $c_{1}^{2}$ and $c_{2}$ the characteristic Chern numbers of $Y$ (also known as $K \cdot K$ and $\chi(Y)$, where $\chi$ is topological Euler characteristic). Then these Chern numbers are determined by the resolution dual graph, and their sum $c_{1}^{2}+c_{2}$ is independent of the resolution, hence depends only on the link. We define

$$
C(\Delta)=c_{1}^{2}+c_{2}-1,
$$

the notation indicating that this number depends only on the splice diagram $\Delta$. Then the aforementioned formulas may be written

$$
\begin{aligned}
\mu & =12 p_{g}+C(\Delta) & & \text { (Laufer) } \\
3 \operatorname{sign}(F) & =-2 \mu-C(\Delta) & & \text { (Durfee) }
\end{aligned}
$$


(In general Durfee's formula has an extra $3 b_{1}(Y)$ on the right, which vanishes in our case.) Eliminating $\mu$, these formulas imply

$$
\operatorname{sign}(F)=-8 p_{g}-C(\Delta),
$$

proving the equivalence of the two formulations of the Casson Invariant Conjecture in the Introduction (for complete intersections). Moreover, it follows that the equivalence of (2), (3), and (4) of Theorem 6.3 reduce to the formula of the following theorem, which will therefore complete the proof.

Theorem 6.4 In the above notation, even if $\Delta$ does not satisfy the semigroup condition we have

$$
C(\Delta)-C\left(\Delta_{1}\right)-C\left(\Delta_{2}\right)=-2 b_{1}\left(G_{1}\right) b_{1}\left(G_{2}\right) .
$$

This theorem involves computing $c_{1}^{2}$ and $c_{2}$ of the resolution in terms of the splice diagram, which is of interest in its own right, so we devote the next section (Section 17) to its proof.

If any one of the analytic invariants $\operatorname{sign}(F), \mu$, and $p_{g}(X)$ is a topological invariant, then they all are, by the above formulas. The Casson Invariant Conjecture gives a topological description of $p_{g}$ and $\operatorname{sign}(F)$.

Suppose $(X, o)$ is a complete intersection surface singularity whose homology sphere link has one node; thus, its link is $\Sigma\left(p_{1}, \cdots, p_{n}\right)$. The Casson Invariant Conjecture for $X$ is equivalent to the assertion that $p_{g}(X)=p_{g}\left(V\left(p_{1}, \cdots, p_{n}\right)\right)$. But this latter condition is well-known to be equivalent to the statement that $X$ admits an equisingular, simultaneous resolution degeneration to $V$ (see, eg, [33] (6.3) for a convenient proof). In other words, we could conclude that $X$ is a splice type singularity, as was mentioned in the Introduction. We suspect a similar result is true in the general case. But, even in case $X$ is a hypersurface singularity with link $\Sigma(p, q, r)$, we do not know a proof. As we mentioned in the Introduction, there are a few very non-trivial cases worked out by A Némethi [19.

\section{Canonical divisor of a resolution}

This section is devoted to proving Theorem 6.4. We start by computing the rational canonical divisor for an arbitrary resolution of an isolated surface singularity. 
Suppose we have a good resolution of an isolated surface singularity. Denote the exceptional curves by $E_{i}, i=1, \ldots, n$. For each $i$ let $\delta_{i}$ be the number of intersection points of $E_{i}$ with other $E_{j}$ 's and let $E_{i}^{0}$ be $E_{i}$ with these intersection points removed. Denote $\chi_{i}=\chi\left(E_{i}^{0}\right)=\chi\left(E_{i}\right)-\delta_{i}$ ( $\chi$ is Euler characteristic).

Let $K$ be the (rational) canonical divisor, defined by the adjunction formula

$$
K \cdot E_{i}=-\chi\left(E_{i}\right)-E_{i} \cdot E_{i}
$$

Let

$$
D:=-K-E, \quad \text { where } E=\sum_{i=1}^{n} E_{i}
$$

and suppose

$$
D=\sum_{i=1}^{n} k_{i} E_{i}
$$

Then the adjunction formula becomes $D \cdot E_{j}=\chi\left(E_{j}\right)-\delta_{j}=\chi_{j}$, so

$$
k_{i}=-\sum \ell_{i j} \chi_{j}, \quad \text { where }\left(\ell_{i j}\right)=\left(-E_{i} \cdot E_{j}\right)^{-1} \text { (matrix inverse). }
$$

Now

$$
K=-D-E=\sum_{i}\left(-k_{i}-1\right) E_{i}=\sum_{i}\left(\sum_{j} \ell_{i j} \chi_{j}-1\right) E_{i}
$$

so

$$
\begin{aligned}
K \cdot K= & \left(\sum_{i}\left(\sum_{j} \ell_{i j} \chi_{j}-1\right) E_{i}\right) \cdot\left(\sum_{k}\left(\sum_{l} \ell_{k l} \chi_{l}-1\right) E_{k}\right) \\
= & \sum_{i, j, k, l} \ell_{i j} \ell_{k l}\left(E_{i} \cdot E_{k}\right) \chi_{j} \chi_{l}-\sum_{i, k, l} \ell_{k l}\left(E_{i} \cdot E_{k}\right) \chi_{l}-\sum_{i, j, k} \ell_{i j}\left(E_{i} \cdot E_{k}\right) \chi_{j} \\
& \quad+\sum_{i, k} E_{i} \cdot E_{k} \\
= & -\sum_{j, l} \ell_{j l} \chi_{j} \chi_{l}+\sum_{i} \chi_{i}+\sum_{i} \chi_{i}+\left(\sum_{i} E_{i} \cdot E_{i}+2 \sum_{i<j} E_{i} \cdot E_{j}\right) \\
= & -\sum_{i, j} \ell_{i j} \chi_{i} \chi_{j}+2 \chi\left(\bigcup_{i} E_{i}\right)+\sum_{i} E_{i} \cdot E_{i} .
\end{aligned}
$$

We note that our notation $k_{i}$ and $\ell_{i j}$ is consistent with the notation in the Appendix (Section 9). Summarizing:

Proposition 7.1 For any good resolution of an isolated surface singularity the divisor $D=-K-E$ is given by

$$
D=\sum k_{i} E_{i} \quad \text { with } \quad k_{i}=-\sum_{j} \ell_{i j} \chi_{j}
$$


where $\left(\ell_{i j}\right)=\left(-E_{i} \cdot E_{j}\right)^{-1}$ (matrix inverse). Also,

$$
c_{1}^{2}+c_{2}=-\sum_{i, j} \ell_{i j} \chi_{i} \chi_{j}+3 c_{2}+\sum E_{i} \cdot E_{i} .
$$

To apply this to prove Theorem 6.4 we now restrict to the case of a singularity with homology sphere link given by a splice diagram $\Delta$. Then $\chi_{i}=2-\delta_{i}$ which vanishes except at nodes and ends of the plumbing graph, so we only need to know $\ell_{i j}$ when $i$ and $j$ index nodes or ends. By Theorem 9.1 this is as follows. If $i \neq j$ then $\ell_{i j}$ is the product of the splice diagram weights adjacent but not on the path from $i$ to $j$ in $\Delta$. If $i=j$ then:

- If $i$ is a node then $\ell_{i i}$ is the product of weights at that node.

- If $i$ is a leaf adjacent to a node with weights $p_{0}, \ldots, p_{n}$ with $p_{0}$ on the edge to $i$ then $\ell_{i i}=\left\lceil p_{1} \ldots p_{n} / p_{0}\right\rceil$.

A simple matrix calculation shows that $-\ell_{i i}$ is the weight one would have to put on a new vertex attached to vertex $i$ by a new edge, to get an extended plumbing diagram of determinant 0 (this is computed in [7] and gives an alternative proof of the description of $\ell_{i i}$ ).

Now let $C(T)$ denote $c_{1}^{2}+c_{2}-1$ computed for a plumbing graph $T$. We want to compute the effect of splicing on $C$. So suppose that the splice diagram $\Delta$ is the result of splicing diagrams $\Delta_{1}$ and $\Delta_{2}$ and let $T, T_{1}$, and $T_{2}$ be the resolution graphs for these splice diagrams. Let $I_{1}$ and $I_{2}$ be index sets for the nodes and leaves of $T_{1}$ and $T_{2}$ with $0 \in I_{1}$ and $1 \in I_{2}$ representing the leaves at which we splice.

Let $\bar{T}_{1}$ be the result of extending $T_{1}$ at vertex 0 by a vertex with weight $-\ell_{00}\left(T_{1}\right)$ and similarly for $\bar{T}_{2}$. Let $\bar{T}$ be the result of attaching $\bar{T}_{1}$ to $\bar{T}_{2}$ by an edge joining the new vertices. Then in [7] it is shown that $T$ results from $\bar{T}$ by a sequence of $(-1)$-blow-downs of vertices of valency 2 followed by one 0 -absorption. Suppose the number of blow-downs is $r$. Then the blow-downs and 0 -absorption remove $(\mathrm{r}+2)$ vertices, so

$$
c_{2}(T)=c_{2}(\bar{T})-(r+2)=c_{2}\left(T_{1}\right)+c_{2}\left(T_{2}\right)-r-1 .
$$

Moreover each blow-down increases $\sum E_{i} \cdot E_{i}$ by 3 and the 0 -absorption does not change it, so

$$
\sum_{T} E_{i} \cdot E_{i}=\sum_{T_{1}} E_{i} \cdot E_{i}-\ell_{00}+\sum_{T_{2}} E_{i} \cdot E_{i}-\ell_{11}+3 r .
$$


Thus

$$
\begin{aligned}
C(T)= & -\sum_{i, j \in I} \ell_{i j} \chi_{i} \chi_{j}+3 c_{2}(T)+\sum_{T} E_{i} \cdot E_{i}-1 \\
= & -\left(\sum_{i, j \in I_{1}} \ell_{i j} \chi_{i} \chi_{j}-\ell_{00}-2 \sum_{i \in I_{1}-\{0\}} \ell_{0 i} \chi_{i}+\sum_{i, j \in I_{2}} \ell_{i j} \chi_{i} \chi_{j}-\ell_{11}-\right. \\
& \left.\quad-2 \sum_{j \in I_{2}-\{1\}} \ell_{1 j} \chi_{j}+2 \sum_{\substack{i \in I_{1}-\{0\} \\
j \in I_{2}-\{1\}}} \ell_{i j} \chi_{i} \chi_{j}\right) \\
& +3\left(c_{2}\left(T_{1}\right)+c_{2}\left(T_{2}\right)-r-1\right) \\
& +\sum_{T_{1}} E_{i} \cdot E_{i}+\sum_{T_{2}} E_{i} \cdot E_{i}-\ell_{00}-\ell_{11}+3 r-1 \\
= & -\sum_{i, j \in I_{1}} \ell_{i j} \chi_{i} \chi_{j}+2 \sum_{i \in I_{1}-\{0\}} \ell_{0 i} \chi_{i}-\sum_{i, j \in I_{2}} \ell_{i j} \chi_{i} \chi_{j} \\
& +2 \sum_{j \in I_{2}-\{1\}} \ell_{1 j} \chi_{j}-2 \sum_{i \in I_{1}-\{0\}} \ell_{i 0} \chi_{i} \sum_{j \in I_{2}-\{1\}} \ell_{j_{1}} \chi_{j} \\
& +3 c_{2}\left(T_{1}\right)+3 c_{2}\left(T_{2}\right) \\
& +\sum_{T_{1}} E_{i} \cdot E_{i}+\sum_{T_{2}} E_{i} \cdot E_{i}-4
\end{aligned}
$$

where the last equality uses the fact that for $i \in I_{1}-\{0\}$ and $j \in I_{2}-\{1\}$ one has $\ell_{i j}=\ell_{i 0} \ell_{1 j}$.

The above simplifies to

$$
\begin{aligned}
C(T) & =C\left(T_{1}\right)+C\left(T_{2}\right)-2\left(-\sum_{i \in I_{1}-\{0\}} \ell_{0 i} \chi_{i}+1\right)\left(-\sum_{j \in I_{2}-\{1\}} \ell_{1 j} \chi_{j}+1\right) \\
& =C\left(T_{1}\right)+C\left(T_{2}\right)-2 \mu\left(T_{1}, 0\right) \mu\left(T_{2}, 1\right),
\end{aligned}
$$

where $\mu\left(T_{i}, i\right)$ is the Milnor number for the knot represented by vertex $i$ in the homology sphere represented by $T_{i}$, that is, the first Betti number of its fiber (it is a basic result of [7] that $\sum_{i \in I_{1}-\{0\}}\left(\ell_{0 i} \chi_{i}\right)$ is the Euler characteristic of the fiber in question). This completes the proof of Theorem 6.4.

\section{Plane curves and their cyclic covers}

Let $(X, o)$ be a hypersurface singularity at the origin given by an equation in the form $z^{n}+g(x, y)=0$ and suppose that its link is a homology sphere. The Casson Invariant Conjecture was proved in this case in [26] by a somewhat 
subtle calculation. In this section we will show that the Milnor Fiber Conjecture (Conjecture 21) holds for these singularities, giving a more conceptual proof of the Casson Invariant Conjecture in this case. We must first explain how these hypersurface singularities fit the format of equations of splice type. In [26] we point out that if the link of $z^{n}+g(x, y)=0$ is a homology sphere, then $g(x, y)=0$ defines an irreducible plane curve singularity at the origin $o \in \mathbb{C}^{2}$. We therefore need to start by discussing how plane curve singularities in general, and irreducible plane curve singularities in particular, fit into the framework of our conjectures.

\subsection{Non-minimal splice diagrams and plane curve singularities}

Theorem 2.1 gives a general sufficient condition for a knot in a homology sphere to be realizable as the link of a germ $(Y, o) \subset(X, o)$ of a curve cut out by a single equation in a complete intersection surface. This has content also for non-minimal splice diagrams. For example, the splice diagram

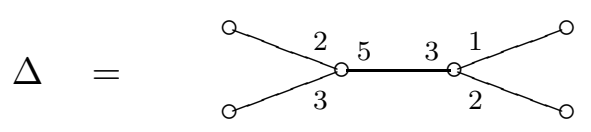

is a non-minimal version of

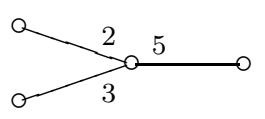

so it represents the Seifert fibered homology sphere $\Sigma(2,3,5)$ (Poincaré's dodecahedral space). The upper right vertex of $\Delta$ represents a particular knot in this homology sphere (a $(3,2)$-cable on the degree 5 fiber of $\Sigma(2,3,5)$ ). Since $\Delta$ satisfies the semigroup condition, Theorem 2.1 tells us that this knot in $\Sigma(2,3,5)$ is the link of a complex curve singularity $(Y, o)$ cut out by a single equation in $(V(2,3,5), o)$. In fact, the splice type equations for $\Delta$ can be chosen as $z_{1}^{2}+z_{2}^{3}+z_{3}^{5}=0, z_{1}+z_{3}^{2}+z_{4}=0$, and the curve is then cut out by $z_{4}=0$. Eliminating $z_{4}$, the curve is cut out by the equation $z_{1}+z_{3}^{2}=0$ in $V(2,3,5)=\left\{\left(z_{1}, z_{2}, z_{3}\right): z_{1}^{2}+z_{2}^{3}+z_{3}^{5}=0\right\}$.

When $X$ is non-singular, that is, for a link of a plane curve singularity, the next proposition implies that we can always do the analogous thing. That is, for any irreducible plane curve singularity we will find strict splice type equations for $X$ $\left(=\mathbb{C}^{2}\right)$ so that the curve $Y$ cut out by a coordinate function has the topology of the given plane curve. Corollary 4.2 then says that the original plane curve 
singularity is a higher weight deformation of the one given by strict splice type equations.

Proposition 8.1 The splice diagram of any plane curve singularity satisfies the semigroup condition.

Proof It is easy to see that the semigroup condition for the splice diagram of a reducible plane curve singularity follows from the semigroup condition for each of the subdiagrams for the irreducible branches of the plane curve. Thus we may assume that the germ $\left(\mathbb{C}^{2}, Y, o\right)$ is an irreducible germ. In this case the result is well known (see, eg, Teissier's appendix to [36]) but we give a proof in our language for completeness. By [7] the singularity is given by a splice diagram of the form:

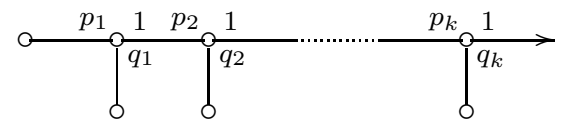

where $\operatorname{gcd}\left(p_{i}, q_{i}\right)=1$ for each $i$ and the positive edge determinant condition holds $\left(p_{i}>q_{i} q_{i-1} p_{i-1}\right.$ for each $\left.i>1\right)$. Since this diagram may have arisen as a subdiagram of a diagram for a plane curve with several branches, we cannot assume that it is a reduced diagram, so some of the $q_{j}$ may equal 1 .

The only non-trivial cases of the semigroup condition for this diagram are:

$$
p_{j+1} \in S_{j}:=\mathbb{N}\left\langle q_{1} q_{2} \ldots q_{j}, p_{1} q_{2} \ldots q_{j}, \ldots, p_{j-1} q_{j}, p_{j}\right\rangle
$$

for each $j=1, \ldots, k-1$. Since $p_{j+1}>p_{j} q_{j} q_{j+1} \geq p_{j} q_{j}$ it suffices to show that the conductor $\mu_{j}$ of this semigroup satisfies $\mu_{j} \leq p_{j} q_{j}$. Proposition 3.3 of section 3 implies $\mu_{j}=q_{j}\left(\mu_{j-1}-1\right)-p_{j}+p_{j} q_{j}+1$ (or use Theorem 2.2 and its following paragraph). The desired inequality is now a trivial induction.

This gives a new way to find an equation for a plane curve singularity of given topology: start with the equations of splice type and then eliminate variables to obtain an equation in $\mathbb{C}^{2}$. To describe this in detail, let us assign variables to the leaves of our splice diagram as follows:

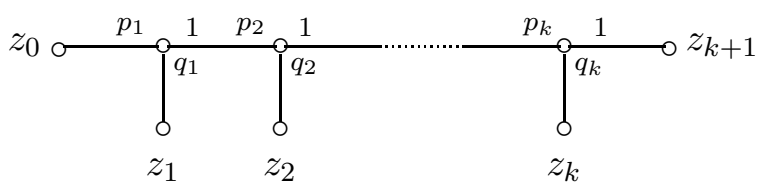

The only admissible monomial for the outgoing edge to the right at the $j$-th node is $z_{j+1}$. Thus the general system of equations of strict splice type can be 
written

$$
\begin{aligned}
z_{2}= & a_{1} z_{1}^{q_{1}}+a_{0} z_{0}^{p_{1}} \\
z_{3}= & a_{2} z_{2}^{q_{2}}+g_{2}\left(z_{0}, z_{1}\right) \\
\ldots & \ldots \quad \ldots \\
z_{k}= & a_{k-1} z_{k-1}^{q_{k-1}}+g_{k-1}\left(z_{0}, \ldots, z_{k-2}\right) \\
z_{k+1}= & a_{k} z_{k}^{q_{k}}+g_{k}\left(z_{0}, \ldots, z_{k-1}\right),
\end{aligned}
$$

where $g_{j}\left(z_{0}, \ldots, z_{j-1}\right)$ is a multiple of an admissible monomial for the left edge at the $j$-th node, that is, a monomial of the form $z_{0}^{\alpha_{0}} \ldots z_{j-1}^{\alpha_{j-1}}$ with

$$
\alpha_{0} q_{1} \ldots q_{j-1}+\alpha_{1} p_{1} q_{2} \ldots q_{j-1}+\cdots+\alpha_{j-2} p_{j-2} q_{j-1}+\alpha_{j-1} p_{j-1}=p_{j} .
$$

We now successively substitute each of the above equations into the next to put them in the form:

$$
\begin{aligned}
z_{2}= & a_{1} z_{1}^{q_{1}}+a_{0} z_{0}^{p_{1}} \\
z_{3}= & a_{2}\left(a_{1} z_{1}^{q_{1}}+a_{0} z_{0}^{p_{1}}\right)^{q_{2}}+g_{2}\left(z_{0}, z_{1}\right)=: f_{2}\left(z_{0}, z_{1}\right) \\
\ldots & \cdots \quad \cdots \\
z_{k+1}= & a_{k} f_{k-1}\left(z_{0}, z_{1}\right)^{q_{k}}+g_{k}\left(z_{0}, z_{1}, \ldots, f_{k-2}\left(z_{0}, z_{1}\right)\right)=: f_{k}\left(z_{0}, z_{1}\right) .
\end{aligned}
$$

In terms of new coordinates, $x:=z_{0}, y:=z_{1}, Z_{2}:=z_{2}-a_{1} z_{1}^{q_{1}}+a_{0} z_{0}^{p_{1}}, \ldots$, $Z_{k}:=z_{k}-f_{k-1}\left(z_{0}, z_{1}\right), Z_{k+1}:=z_{k+1}-f_{k}\left(z_{0}, z_{1}\right)$ these equations become

$$
Z_{2}=Z_{3}=\cdots=Z_{k}=Z_{k+1}=0,
$$

so our surface is the $(x, y)$-plane. Our plane curve is the curve cut out by the coordinate equation $z_{k+1}=0$ which is $f_{k}(x, y)=0$ in our new coordinates. Thus, if we write $f=f_{k}$, the equation of the plane curve is $f(x, y)=0$.

We now address what the Milnor Fiber Conjecture says for this type of example. Our surface germ is a nonsingular point, and the Milnor fiber for a non-singular point is a disk, so the conjecture postulates a particular decomposition of $D^{4}$. Although it is rather trivial, it will be needed in the discussion of hypersurfaces of the form $z^{n}=g(x, y)$. We will therefore reserve the notations $G_{1}$ etc. of Conjecture 2 for that case and use primes (as in $G_{1}^{\prime}$ etc) to distinguish the ingredients involved in the present discussion.

Suppose therefore that we have decomposed our splice diagram as the splice of two diagrams:

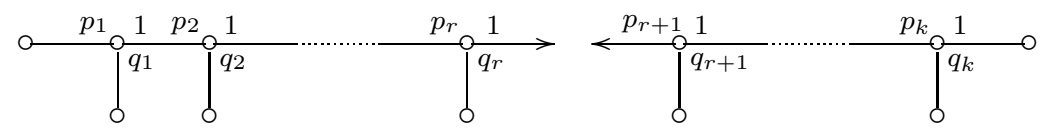


The left diagram represents a plane curve whose Milnor fiber we will denote by $G_{1}^{\prime} \subset S^{3}=\partial D^{4}$. The right diagram is a non-reduced diagram for the trivial knot in $S^{3}$ so its Milnor fiber is $G_{2}^{\prime}=D^{2} \subset S^{3}=\partial D^{4}$.

Let $\left(F_{1}^{\prime}\right)^{o}$ be the result of removing from $D^{4}$ a tubular neighborhood of $G_{1}^{\prime}$ pushed inside to a proper embedding $G_{1}^{\prime} \subset D^{4}$. Let $\left(F_{2}^{\prime}\right)^{o}$ be the result of removing a tubular neighborhood of a proper embedding $D^{2} \subset D^{4}$. Note that $\left(F_{2}^{\prime}\right)^{o} \cong S^{1} \times D^{3}$. The Milnor Fiber Conjecture says that the result of the pasting:

$$
\left(F_{1}^{\prime}\right)^{o} \cup\left(G_{1}^{\prime} \times D^{2}\right) \cup\left(F_{2}^{\prime}\right)^{o}
$$

should be $D^{4}$. This is indeed clear, since, starting with $\left(F_{1}^{\prime}\right)^{o}$, the first pasting clearly gives $D^{4}$ back, while the second just pastes a collar onto a portion of the boundary of this $D^{4}$.

\subsection{The hypersurface $z^{n}+g(x, y)=0$}

As already mentioned, if the link of $z^{n}+g(x, y)=0$ is a homology sphere, then $g(x, y)=0$ defines a plane curve singularity at $(0,0) \in \mathbb{C}^{2}$ which is irreducible. Its splice diagram therefore has the form

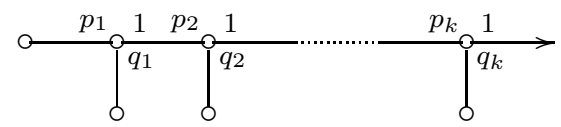

where $\operatorname{gcd}\left(p_{i}, q_{i}\right)=1$ for each $i$ and the positive edge determinant condition holds $\left(p_{i}>q_{i} q_{i-1} p_{i-1}\right.$ for each $\left.i>1\right)$. Moreover, given an irreducible plane curve singularity as above, we showed in 26] that the hypersurface singularity defined by

$$
z^{n}+g(x, y)=0
$$

has homology sphere link if and only if $n$ is relatively prime to all the $p_{i}$ and $q_{i}$, and the splice diagram for the link of this singularity is then:

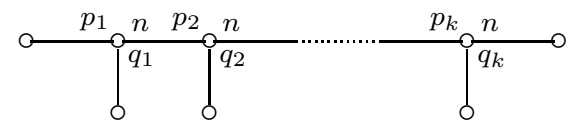

We now show that the splice diagram equations for this splice diagram reduce to the equation $z^{n}=f(x, y)$, with $f$ as in the previous subsection (Corollary 4.2 below shows that the original $z^{n}=g(x, y)$ is an equisingular deformation of this). We assign variables to the leaves of the splice diagram (10) as follows:

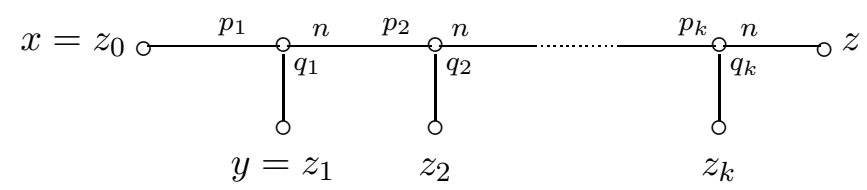


The only admissible monomial for the outgoing edge to the right at the $j$-th node is $z_{j+1}$ if $j<k$ and $z^{n}$ if $j=k$. Thus the general system of equations of strict splice type can be written

$$
\begin{aligned}
z_{2}= & a_{1} z_{1}^{q_{1}}+a_{0} z_{0}^{p_{1}} \\
z_{3}= & a_{2} z_{2}^{q_{2}}+g_{2}\left(z_{0}, z_{1}\right) \\
\ldots & \quad \ldots \quad \ldots \\
z_{k}= & a_{k-1} z_{k-1}^{q_{k-1}}+g_{k-1}\left(z_{0}, \ldots, z_{k-2}\right) \\
z^{n}= & a_{k} z_{k}^{q_{k}}+g_{k}\left(z_{0}, \ldots, z_{k-1}\right),
\end{aligned}
$$

where the $g_{j}\left(z_{0}, \ldots, z_{j-1}\right)$ are as before.

We again successively substitute each of these equations into the next to eliminate the variables $z_{2}, z_{3}, \ldots, z_{k}$. To be precise, we first make these substitutions to put the equations in the form:

$$
\begin{aligned}
z_{2} & =a_{1} z_{1}^{q_{1}}+a_{0} z_{0}^{p_{1}} \\
z_{3} & =f_{2}\left(z_{0}, z_{1}\right) \\
\ldots & \quad \cdots \quad \ldots \\
z_{k} & =f_{k-1}\left(z_{0}, z_{1}\right) \\
z^{n} & =f_{k}\left(z_{0}, z_{1}\right) .
\end{aligned}
$$

Recall our notation $f=f_{k}$. In terms of new coordinates, $x=z_{0}, y=z_{1}, z$, $Z_{2}:=z_{2}-a_{1} z_{1}^{q_{1}}+a_{0} z_{0}^{p_{1}}, \ldots, Z_{k}:=z_{k}-f_{k-1}\left(z_{0}, z_{1}\right)$, these equations become

$$
Z_{2}=Z_{3}=\cdots=Z_{k}=0 ; \quad z^{n}=f(x, y) \text {. }
$$

We are now ready to prove the main result of this section.

Theorem 8.2 Let $(X, o)$ be a hypersurface singularity at the origin given by an equation in the form $z^{n}+g(x, y)=0$ with homology sphere link. Then the Milnor Fiber Conjecture is true for $(X, o)$.

Proof Suppose that we have a splice decomposition corresponding to the following decomposition of our splice diagram as the splice of two diagrams:

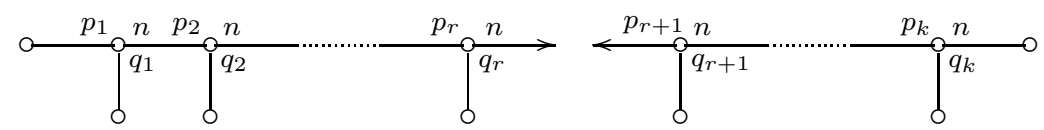

We wish to show that the Milnor fiber $F$ for $z^{n}=g(x, y)$ is obtained by the construction $F_{1}^{o} \cup_{N_{1}}\left(G_{1} \times G_{2}\right) \cup_{N_{2}} F_{2}^{o}$ of Conjecture 2, where $F_{1}$ and $F_{2}$ are Milnor fibers for the two splice components, $G_{1}$ and $G_{2}$ are fibers in the links 
of the two splice components for the knots along which we splice, and $F_{i}^{o}$ is the result of removing a tubular neighborhood of a properly embedded $G_{i}$ in $F_{i}$.

In [24] (see also [12]) it is shown that the Milnor fiber $F$ is obtained by taking a Milnor fiber $G \subset S^{3}=\partial D^{4}$ for $g$, pushing it inside $D^{4}$ so that it is properly embedded (that is, $\partial G=G \cap \partial D^{4}$ ), and then taking the $n$-fold branched cyclic cover of $D^{4}$, branched along this embedding of $G$.

We need to understand the placement of $G$ with respect to the decomposition of $D^{4}$ of equation (9). On taking the n-fold branched cover we will see that we get the desired decomposition of $F$.

According to 7 the fiber $G$ decomposes according to the splice diagram into $q_{r+1} \ldots q_{k}$ parallel copies of the Milnor fiber $G_{1}^{\prime}$ of the plane curve given by

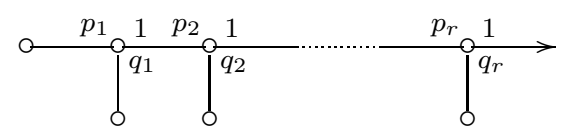

and one copy of the Milnor fiber of the plane curve corresponding to

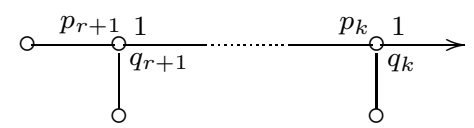

punctured $q_{r+1} \ldots q_{k}$ times.

We can position $G$ with respect to the decomposition of equation (9) so that it lies completely in $\left(G_{1}^{\prime} \times D^{2}\right) \cup\left(F_{2}^{\prime}\right)^{o}$. It then intersects $\left(G_{1}^{\prime} \times D^{2}\right)$ in $q_{r+1} \ldots q_{k}$ parallel copies of $G_{1}^{\prime}$. Its intersection with $\left(F_{2}^{\prime}\right)^{o}$ is obtained as follows. First make the fiber $G^{\prime}$ of the knot represented by the right arrowhead of the splice diagram:

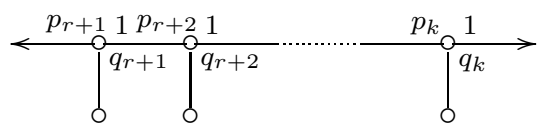

properly embedded in $D^{4}$ and transverse to the properly embedded version of the fiber $D^{2}$ of the unknot represented by the left arrowhead. Then remove the tubular neighborhood of the latter. Using [24], the $n$-fold cyclic cover of $D^{4}$ along $G^{\prime}$ is the Milnor fiber for the surface singularity with diagram:

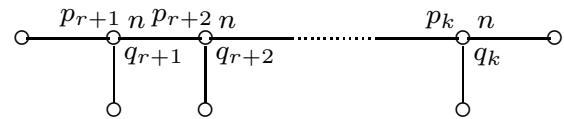

Moreover, the embedded $D^{2} \subset D^{4}$ lifts in this cover to copy of the fiber for the knot represented by the left-most vertex.

It follows that the decomposition of equation (9) lifts to give the desired decomposition of $F$, as desired. 
In the context of the above result it is worth mentioning that Némethi and Mendris recently showed [20] that for a singularity $z^{n}=f(x, y)$ with homology sphere link (even rational homology sphere link) the Milnor fibration is topologically determined by the link of the singularity.

Remark 8.3 The results of this section give a proof of the Casson Invariant Conjecture (CIC) for these examples, also proven in [26, 5, 23. Saveliev and Collin [5], using equivariant Casson invariant, give an iterative generalization of these examples but their approach implies more: Let $\Delta$ be any splice diagram satisfying the semigroup condition and $w$ a leaf of $\Delta$. We allow, as in this section, the weight on the edge to $w$ to be 1 . For $n \in \mathbb{N}$ let $\Delta_{n}(w)$ be the diagram obtained by multiplying the weight furthest from $w$ on each edge by $n$. We assume $n$ is chosen coprime to all the unchanged weights at each node, so $\Delta_{n}(w)$ is again a splice diagram. Then if CIC is valid for splice type singularities for $\Delta$, then the same holds for $\Delta_{n}(w)$.

\section{Appendix: Splicing and plumbing}

In this appendix we recall the classification of $\mathbb{Z}$-homology sphere singularity links in terms of splice diagrams and describe how to recover a resolution diagram from the splice diagram.

We start with Seifert fibered manifolds. For the following results see [27]. Let $\Sigma$ be a Seifert fibered homology 3-sphere other than $S^{3}$. Then it has at least 3 singular fibers and the degrees $p_{1}, \ldots, p_{r}$ of these singular fibers are pairwise coprime. Conversely, given a set $\left\{p_{1}, \ldots, p_{r}\right\}$ of pairwise coprime integers $p_{i}>1$ with $r \geq 3$, there is a unique Seifert fibered homology sphere $\Sigma\left(p_{1}, \ldots, p_{r}\right)$ up to orientation with these singular fiber degrees. Moreover, $\Sigma\left(p_{1}, \ldots, p_{r}\right)$ has a unique orientation for which it is a singularity link, so we give it this orientation. It is, in fact, the link of the Brieskorn complete intersection

$V\left(p_{1}, \ldots, p_{r}\right):=\left\{\left(z_{1}, \ldots, z_{r}\right) \in \mathbb{C}^{n}: a_{i 1} z_{1}^{p_{1}}+\cdots+a_{i r} z_{r}^{p_{r}}=0\right.$ for $\left.i=1, \ldots, r-2\right\}$,

for a sufficiently general matrix $\left(a_{i j}\right)$ of coefficients. By Hamm [9], "sufficiently general" means that all $(r-2) \times(r-2)$ minors should be non-singular.

We represent the homology sphere $\Sigma\left(p_{1}, \ldots, p_{r}\right)$ by the splice diagram:

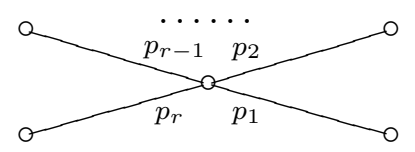


Each of the singular fibers of $\Sigma\left(p_{1}, \ldots, p_{r}\right)$ represents a knot in $\Sigma\left(p_{1}, \ldots, p_{r}\right)$ which we represent in a splice diagram by adding an arrowhead to the corresponding edge. Thus

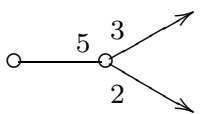

represents the link in $\Sigma(2,3,5)$ consisting of the degree 2 and 3 singular fibers. Non-singular fibers are represented by adding new arrows at the central vertex weighted by 1 , so

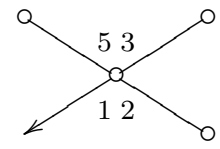

represents the knot in $\Sigma(2,3,5)$ consisting of one non-singular fiber.

There are Seifert fibrations of the 3 -sphere with 2 or less singular fibers. For instance, $S^{3}$ can be fibered by copies of the $(p, q)$ torus knot, with one $p$-fold singular fiber and one $q$-fold singular fiber, so the splice diagram

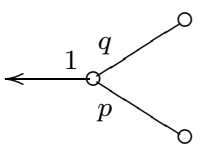

is the diagram for the $(p, q)$ torus knot in $S^{3}$. Similarly

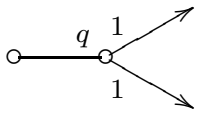

represents a pair of parallel $(1, q)$ torus knots (unknotted curves which link each other $q$ times).

If $K_{1} \subset \Sigma_{1}$ is a knot in a homology sphere and $K_{2} \subset \Sigma_{2}$ is another, then we form the splice of $\Sigma_{1}$ to $\Sigma_{2}$ along $K_{1}$ and $K_{2}$ as follows. Let $N_{i}$ be a closed tubular neighborhood of $K_{i}$ in $\Sigma_{i}$ for $i=1,2$ and let $\Sigma_{i}^{\prime}$ be the result of removing its interior, so $\partial \Sigma_{i}^{\prime}=T^{2}$. The splice is the manifold

$$
\Sigma=\Sigma_{1}^{\prime} \cup_{T^{2}} \Sigma_{2}^{\prime},
$$

where the gluing matches meridian in $\Sigma_{1}$ to longitude in $\Sigma_{2}$ and vice versa. ("Meridian" and "longitude" in $\Sigma_{1}^{\prime}$ are the simple curves in $\partial \Sigma_{1}^{\prime}=T^{2}$ that are null-homologous respectively in the removed solid torus $N_{1}$ or in $\Sigma_{1}^{\prime}$.) We denote the splice by

$$
\Sigma=\Sigma_{1} \underline{K_{1} \quad K_{2}} \Sigma_{2}
$$


We represent splicing in terms of splice diagrams by gluing the diagrams at the arrowheads that represent the knots along which we are splicing. For instance,

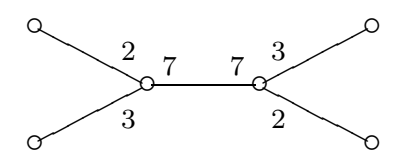

represents the splice of two copies of $\Sigma(2,3,7)$ along the knots represented by the degree 7 fibers.

By [7], the splice diagrams that classify homology sphere singularity links are precisely the splice diagrams with pairwise coprime positive weights around each node and with positive edge determinants (recall that the edge determinant is the product of the two weights on the edge minus the product of the weights adjacent to the edge).

The splice diagram can be computed very easily from a resolution diagram for the singularity. We describe this in detail in the appendix of [30] so we will not repeat it here. Briefly, the splice diagram is obtained from the dual resolution graph for the singularity by replacing each string in the resolution graph by a single edge (ie, we eliminate vertices of valence 2 ); each splice diagram weight is the absolute value of the determinant of the intersection matrix for the subgraph of the resolution graph cut off at the corresponding node in the direction of the corresponding edge.

We will also need an "unreduced" version of the splice diagram: The maximal splice diagram is the version of the splice diagram we get from the resolution graph if we do not first eliminate vertices of valency 2 , and we include edge weights at all vertices - also the leaves. For example, the resolution graph

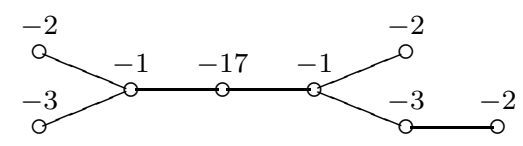

gives maximal splice diagram and splice diagram

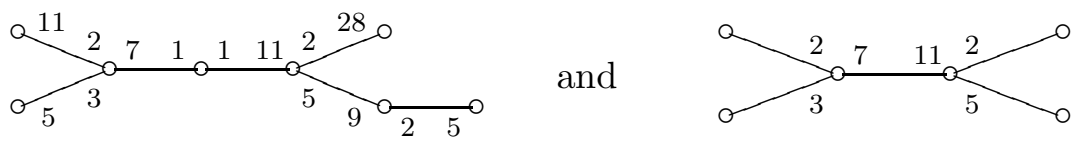

respectively (this is Example 12.1 in [30]).

An algorithm to recover the resolution diagram from the splice diagram is given in [7. Here we describe an easier method that arose from conversations with Paul Norbury (developed independently by Pierrette Cassou-Nogues [3], whose 
terminology of "maximal splice diagram" we have adopted - we called it "adjoint diagram").

To compute the resolution graph from the splice diagram we will give algorithms to:

- compute the maximal splice diagram from the splice diagram, and

- compute the resolution graph from the maximal splice diagram.

We will need the following properties of the maximal splice diagram, which are proved in greater generality in section 12 of [30] (Theorem 12.2 and Lemma 12.5).

Theorem 9.1 (1) For any pair of vertices $v$ and $w$ of the maximal diagram let $\ell_{v w}$ be the product of the weights adjacent to, but not on, the shortest path from $v$ to $w$ in $\Delta^{\prime}$. Then the matrix $L:=\left(\ell_{v w}\right)$ is the inverse matrix of $-A(T)$.

(2) Every edge determinant for the maximal splice diagram is 1.

(3) The edge-weight adjacent to a leaf $v$ of the maximal splice diagram is equal to $\lceil a / b\rceil$ where $a$ is the product of edge-weights adjacent to and just beyond the nearest node to $v$ and $b$ is the remaining weight adjacent to that node.

We remark that part (3) is valid also for the valency 2 vertices between the leaf and its nearest node. For example, for the right-most leaf of the above example $5=\lceil 22 / 5\rceil=\lceil 9 / 2\rceil$.

\subsection{Maximal splice diagram from splice diagram}

We describe how to recover the string of vertices and weights of the maximal splice diagram between any two vertices of a splice diagram. Suppose first both vertices are nodes with weights as follows,

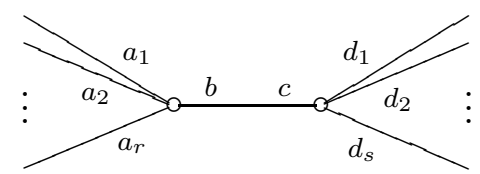

and put $a=\prod_{1}^{r} a_{i}, d=\prod_{1}^{s} d_{j}$. If one of the vertices (say the right one) is a leaf instead of a node then we put $d=1$. The desired string of vertices and weights between our two nodes will only depend on $a, b, c, d$, so we replace the above diagram by:

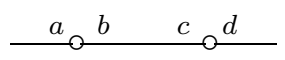


Consider the following infinite linear graph:

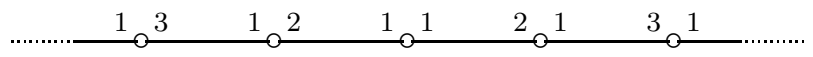

We are going to refine this by adding vertices on this line until our vertices $\stackrel{a}{a} b$ and $\stackrel{c_{0} d}{a}$ appear on it. Vertices $x_{0}^{y}$ are ordered along the line by size of $x / y$. Thus such a vertex either is already a vertex of the linear graph, or it falls on an existing edge. In the latter case we subdivide the edge as follows:

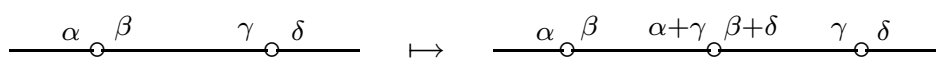

We repeat this process until both our desired vertices appear, and then the portion of the linear graph between them is what we were seeking.

For example, suppose our initial splice diagram is:

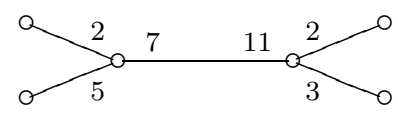

To create the string for the middle edge we start with:

$$
10 \sim 7 \quad 11 م 6
$$

and apply the above procedure. We mark the positions of these vertices, until they are found, by $\vee$.

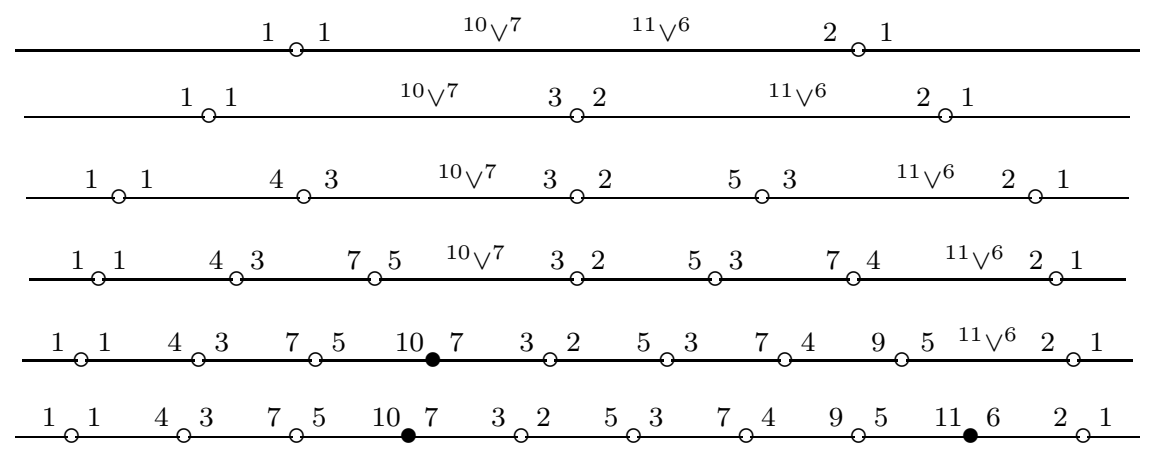

Thus the final string is:

\begin{tabular}{|c|c|c|c|}
\hline $7 \quad 1$ & ${ }^{22} \vee^{3}$ & & 8.1 \\
\hline 71 & ${ }^{22} \vee^{3}$ & 152 & 81 \\
\hline 71 & 223 & 152 & 81 \\
\hline
\end{tabular}

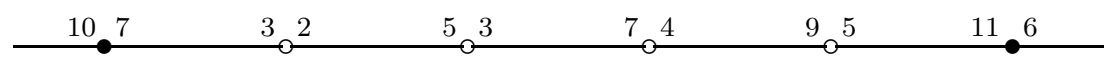

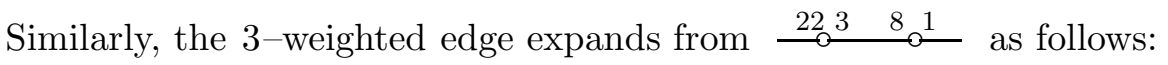


A shortcut is available in the above procedure: to compute the string for the central edge we did not need to create the $(4,3)$ - and $(7,5)$-vertices, since the edge determinant on the edge from $(10,7)$ to $(3,2)$ is already 1.

With this comment, the other three edges are immediate and the maximal splice diagram is:

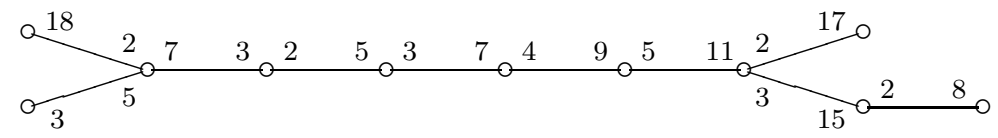

\subsection{Resolution graph from maximal splice diagram}

We must recover the self-intersection weights $e_{v}:=a_{v v}$ at vertices. The matrix equation $L A(T)=-I$ gives equations that will do this. We use the notation $w-v$ to mean vertices $w$ and $v$ are connected by an edge. Then for any vertex $w^{\prime}$ adjacent to $v$, the $v w^{\prime}$ entry of this matrix equation gives:

$$
e_{v}=\frac{-1}{\ell_{v w^{\prime}}}\left(\sum_{\{w: w-v\}} \ell_{w w^{\prime}}\right)
$$

Note that the product of the weights just beyond $w^{\prime}$ from $v$ cancel in this formula, so they may be replaced by 1 for the calculation. For example, for the above maximal splice diagram we get the resolution graph:

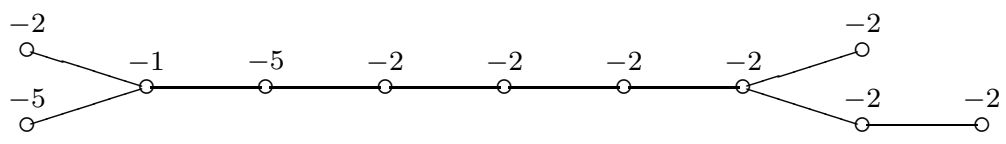

\subsection{Proof of the procedure of 9.1}

The procedure in [7] implies that the string of the maximal splice diagram between the two vertices in question only depends on $a, b, c, d$. Consider the resolution graph

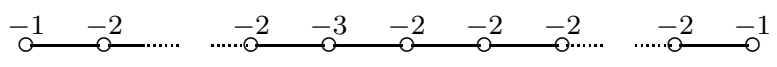

with associated maximal splice diagram:

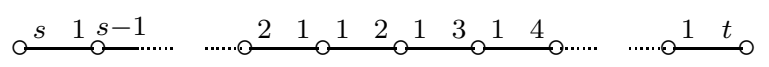

This is a piece of the infinite linear graph we used above, and we choose $s$ and $t$ large enough that our desired vertices will lie in this piece. Now we repeatedly 
blow up on edges of the linear resolution graph. An easy calculation shows that blowing up on an edge:

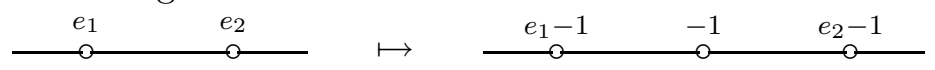

has the effect:

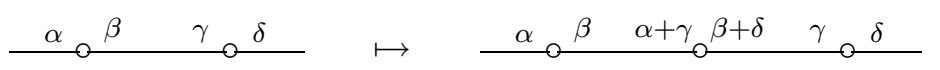

on the associated maximal splice diagram. Thus we need only show that our desired vertices eventually appear in this procedure. But this is a standard fact about Farey sequences (alternatively, one can observe that we are describing the standard procedure to resolve the plane curve singularity $\left.\left(x^{a}+y^{b}\right)\left(x^{c}+y^{d}\right)\right)$.

This same argument applies to see how to fill in the maximal splice diagram between a node and a leaf, even if the edge weight at the leaf is unknown. The leaf will be the rightmost vertex of the above string (12) with $t$ chosen as small as possible to accommodate our desired vertex $\stackrel{a_{0} b}{b}$. Thus, the $t$ that we choose is $\lceil a / b\rceil$ (if $t=1$ the initial resolution string (11) is $\begin{array}{ccc}-1 & -2 & -2 \\ 0\end{array}$

Note that this blow-up procedure gives an alternative way to compute the selfintersection weights along the string, making the calculation of subsection 9.2 only necessary at nodes.

\section{References}

[1] W Barth, C Peters, A Van de Ven, Compact Complex Surfaces, Ergebnisse der Mathematik und ihre Grenzgebiete (3) 4, Springer-Verlag (1984) MathReview

[2] R-O Buchweitz, G-M Greuel, The Milnor number and deformations of complex curve singularities, Invent. Math. 58 (1980) 241-281 MathReview

[3] P Cassou-Nogués, A Płoski, Introduction to Algebraic Plane Curve Singularities, in preparation

[4] O Collin, Equivariant Casson invariant for knots and the Neumann-Wahl formula, Osaka J. Math. 37 (2000) 57-71 MathReview

[5] O Collin, N Saveliev, Equivariant Casson invariants via gauge theory, J. Reine Angew. Math. 541 (2001) 143-169 MathReview

[6] C Delorme, Sous-monö̈des d'intersection complète de $\mathbb{N}$, Ann. Sci. École Norm. Sup. (4) 9 (1976) 145-154 MathReview

[7] D Eisenbud, W D Neumann, Three-dimensional link theory and invariants of plane curve singularities, Ann. Math. Stud. 110, Princeton Univ. Press (1985) MathReview 
[8] J Giraud, Improvement of Grauert-Riemenschneider's theorem for a normal surface, Ann. Inst. Fourier (Grenoble) 32 (1982) 13-23 (1983) MathReview

[9] H A Hamm, Exotische Sphären als Umgebungsränder in speziellen komplexen Räumen, Math. Ann. 197 (1972) 44-56 MathReview

[10] J Herzog, Generators and relations of abelian semigroups and semigroup rings, Manuscripta Math, 3 (1970) 175-193 MathReview

[11] J Herzog, E Kunz, Die Wertehalbgruppe eines lokalen Rings der Dimension 1, Sitzungsberichte der Heidelberger Akademie der Wissenschaften, 2. Abh. (1971, Springer Verlag) 27-67 MathReview

[12] L Kauffman, W D Neumann, Product of knots, branched fibrations, and sums of singularities, Topology 16 (1977) 369-393 MathReview

[13] H Laufer, On generalized Weierstrass points and rings with no prime elements, from: "Riemann surfaces and related topics: Proceedings of the 1978 Stony Brook Conference", Ann. of Math. Stud. 97 Princeton Univ. Press (1981) 391402 MathReview

[14] Y Lim, The equivalence of Seiberg-Witten and Casson invariants for homology 3-spheres, Math. Res. Lett. 6 (1999) 631-643 MathReview

[15] C Lescop, Invariant de Casson-Walker des sphères d'homologie rationnelle fibrés de Seifert, C. R. Acad. Sci. Paris Sér. I Math. 310 (1990) 727-730 MathReview

[16] C Lescop, Global surgery formula for the Casson-Walker invariant. Ann. of Math. Stud. 140 Princeton Univ. Press (1996) MathReview

[17] I Luengo-Velasco, A Melle-Hernandez, A Némethi, Links and analytic invariants of superisolated singularities, to appear in J. Alg. Geom. arXiv:math.AG/0312416

[18] J Milnor, Singular points of complex hypersurfaces, Annals of Mathematics Studies, 61, Princeton University Press, Princeton, N.J.; University of Tokyo Press, Tokyo (1968) MathReview

[19] A Némethi, Weakly elliptic Gorenstein singularities of surfaces, Invent. Math. 137 (1999) 145-167 MathReview

[20] A Némethi, $\mathbf{R}$ Mendris, The link of $f(x, y)+z^{n}=0$ and Zariski's Conjecture, arXiv:math.AG/0207212

[21] A Némethi, L E Nicolaescu, Seiberg-Witten invariants and surface singularities, Geom. Topol. 6 (2002) 269-328 MathReview

[22] A Némethi, LE Nicolaescu, Seiberg-Witten invariants and surface singularities II. Singularities with good $\mathbb{C}^{*}$-action, J. London Math. Soc. 69 (2004) 593-607 MathReview

[23] A Némethi, L E Nicolaescu, Seiberg-Witten invariants and surface singularities III. Splicings and cyclic covers, arXiv:math.AG/0207018 
[24] W D Neumann, Cyclic suspension of knots and periodicity of signature for singularities, Bull. Amer. Math. Soc. 80 (1974) 977-982 MathReview

[25] W D Neumann, A calculus for plumbing applied to the topology of complex surface singularities and degenerating complex curves, Trans. Amer. Math. Soc. 268 (1981) 299-343 MathReview

[26] W D Neumann, J Wahl, Casson invariant of links of singularities, Comment. Math. Helv. 65 (1990) 58-78 MathReview

[27] W D Neumann, F Raymond, Seifert manifolds, plumbing, $\mu$-invariant, and orientation reversing maps, from: "Algebraic and Geometric Topology", Lecture Notes in Math. 664, Springer-Verlag (1978) 162-194 MathReview

[28] W D Neumann, J Wahl, Universal abelian covers of surface singularities, from: "Trends in Singularities", (A Libgober and M Tibar, editors) Trends Math. Birkhäuser (2002) 181-190 MathReview

[29] W D Neumann, J Wahl, Universal abelian covers of quotient-cusps, Math. Ann. 326 (2003) 75-93 MathReview

[30] W D Neumann, J Wahl, Complete intersections singularities of splice type as universal abelian covers, Geom. Topol. 9 (2005) 699-755

[31] L Siebenmann, On vanishing of the Rohlin invariant and nonfinitely amphicheiral homology 3 -spheres, from: "Topology Symposium, Siegen 1979, Proc. Sympos. Univ. Siegen, Siegen, 1979", Lecture Notes in Math., 788 SpringerVerlag (1980) 172-222 MathReview

[32] B Teissier, Introduction to curve singularities, from: "Singularity theory (Trieste, 1991)", World Sci. Publishing, River Edge, NJ (1995) 866-893 MathReview

[33] M Tomari, K Watanabe, Filtered rings filtered blowing-ups and normal twodimensional singularities with "star-shaped" resolution, Publ. Res. Inst. Math. Sci. 25 (1989) 681-740 MathReview

[34] J Wahl, Smoothings of normal surface singularities, Topology 20 (1981) 219246 MathReview

[35] K Watanabe, Some examples of one dimensional Gorenstein domains, Nagoya Math. J. 49 (1973) 101-109 MathReview

[36] O Zariski, Le problème des modules pour les branches planes, École Polytechnique, Paris (1973) MathReview 